UDC 512.542

\title{
Carter subgroups of finite almost simple groups
}

\author{
E.P.Vdovin
}

\begin{abstract}
Annotation
In the paper we complete the classification of Carter subgroups in finite almost simple groups. In particular, we prove that Carter subgroups of every finite almost simple group are conjugate. Togeather with previous results by author and F. Dalla Volta, A. Lucchini, and M. C. Tamburini, as a corollary, it follows that Carter subgroups of every finite group are conjugate.
\end{abstract}

\section{Introduction}

We recall that a subgroup of a finite group is called a Carter subgroup if it is nilpotent and selfnormalizing. By a well-known result, any finite solvable group contains exactly one conjugacy class of Carter subgroups (cf. [1]), and it is reasonable to conjecture that a finite group contains at most one conjugacy class of Carter subgroups. The evidence for this conjecture is based on extensive investigation, by several authors, of classes of finite groups which are close to be simple. In particular it has been shown that the conjecture holds for the symmetric and alternating groups (cf. [2]) and, denoting by $p^{t}$ a power of a prime $p$, for any group $A$ such that $S L_{n}\left(p^{t}\right) \leq A \leq G L_{n}\left(p^{t}\right)$ (cf. [3] and [4]), for the symplectic groups $S p_{2 n}\left(p^{t}\right)$, the full unitary groups $G U_{n}\left(p^{2 t}\right)$ and, when $p$ is odd, the full orthogonal groups $G O_{n}^{ \pm}\left(p^{t}\right)$ (cf. [5]). Later in [6] results of [5] were extended to any group $G$ with $O^{p^{\prime}}(S) \leq G \leq S$, where $S$ is a full classical matrix group. Also some of the sporadic simple groups were investigated (cf. [7], for example). In the nonsolvable cases, when Carter subgroups exist, they always turn out to be the normalizers of Sylow 2-subgroups.

In the paper we consider the following

Problem. Are any two Carter subgroups of a finite group conjugate?

In [8] it is proven that the minimal counterexample $A$ to this problem should be almost simple. Later in [9] a stronger result was obtained. A finite group $G$ is said to satisfy condition $(*)$ if, for every its non-Abelian composition factor $S$ and for every its nilpotent $\operatorname{subgroup~} N$, Carter subgroups of $\left\langle\operatorname{Aut}_{N}(S), S\right\rangle$ are conjugate (definition of $\operatorname{Aut}_{N}(S)$ one can find below). In [9] the following theorem was proven.

Theorem 1.1. If a finite group $G$ satisfies $(*)$, then Carter subgroups of $G$ are conjugate.

Thus our goal here is to prove that for every known simple group $S$ and every nilpotent subgroup $N$ of $\operatorname{Aut}(S)$, Carter subgroups of $\langle S, N\rangle$ are conjugate. Some classes of almost simple groups which can not be minimal counter example to the problem are found in [6] and [10]. The resulting table of almost simple groups with conjugate Carter subgroups is given in [9].

Our notations is standard. If $G$ is a finite group, we denote by $\mathbf{P} G$ the factor group $G / Z(G)$. If $\pi$ is a set of primes then we denote by $\pi^{\prime}$ its complement in the set of all primes. As usual we denote by $O_{\pi}(G)$ the maximal normal $\pi$-subgroup of $G$ and we denote by $O^{\pi^{\prime}}(G)$ the subgroup generated by all $\pi$-elements of $G$. If $\pi=\{2\}^{\prime}$ is the set of all odd primes, then $O_{\pi}(G)=O_{2^{\prime}}(G)$ is denoted by $O(G)$. If $g \in G$, then we denote by $g_{\pi}$ the $\pi$-part of $g$, i. e., $g_{\pi}=g^{|g|_{\pi^{\prime}}}$. For a 
finite group $G$ we denote by $\operatorname{Aut}(G)$ the group of automorphisms of $G$. If $\lambda \in \operatorname{Aut}(G)$, then we denote by $G_{\lambda}$ the set of $\lambda$-stable points, i. e., $G_{\lambda}=\left\{g \in G \mid g^{\lambda}=g\right\}$. If $Z(G)$ is trivial, then $G$ is isomorphic to the group of its inner automorphisms and we may suppose that $G \leq \operatorname{Aut}(G)$. A finite group $G$ is said to be almost simple if there is a simple group $S$ with $S \leq G \leq \operatorname{Aut}(S)$, i. e., $F^{*}(G)$ is a simple group. We denote by $F(G)$ the Fitting subgroup of $G$ and by $F^{*}(G)$ the generalized Fitting subgroup of $G$.

If $G$ is a group, $A, B, H$ are subgroups of $G$ and $B$ is normal in $A(B \unlhd A)$, then $N_{H}(A / B)=$ $N_{H}(A) \cap N_{H}(B)$. If $x \in N_{H}(A / B)$, then $x$ induces an automorphism $B a \mapsto B x^{-1} a x$ of $A / B$. Thus, there is a homomorphism of $N_{H}(A / B)$ into $\operatorname{Aut}(A / B)$. The image of this homomorphism is denoted by $\operatorname{Aut}_{H}(A / B)$ while its kernel is denoted by $C_{H}(A / B)$. In particular, if $S$ is a composition factor of $G$, then for any $H \leq G$ the $\operatorname{group}^{\operatorname{Aut}_{H}}(S)$ is defined.

\section{$2 \quad$ Preliminary results}

Lemma 2.1. Let $G$ be a finite group, let $K$ be a Carter subgroup of $G$ and assume that $N$ is a normal subgroup of $G$. Assume that $K N$ satisfies $(*)$. Then $K N / N$ is a Carter subgroup of $G / N$.

Proof. Consider $x \in G$ and assume that $x N \leq N_{G / N}(K N / N)$. It follows that $x \in N_{G}(K N)$. We have that $K^{x}$ is a Carter subgroup of $K N$. Since $K N$ satisfies (*), we have that its Carter subgroups are conjugate. Thus there exists $y \in K N$ such that $K^{y}=K^{x}$. Since $K$ is a Carter subgroup of $G$, it follows that $x y^{-1} \in N_{G}(K)=K$ and $x \in K N$.

Lemma 2.2. [9, Lemma 5] Assume that $G$ is a finite group. Let $K$ be a Carter subgroup of $G$, with centre $Z(K)$. Assume also that $e \neq z \in Z(K)$ and $C_{G}(z)$ satisfies $(*)$.

(1) Every subgroup $Y$ which contains $K$ and satisfies $(*)$ is self-normalizing in $G$.

(2) No conjugate of $z$ in $G$, except $z$, lies in $Z(K)$.

(3) If $H$ is a Carter subgroup of $G$, non-conjugate to $K$, then $z$ is not conjugate to any element in the centre of $H$.

In particular the centralizer $C_{G}(z)$ is self-normalizing in $G$, and $z$ is not conjugate to any power $z^{k} \neq z$.

Lemma 2.3. Let $G$ be a finite group and $S$ be a Sylow 2-subgroup of $G$. Then $G$ contains a Carter subgroup $K$ with $S \leq K$ if and only if $N_{G}(S)=S C_{G}(S)$.

Proof. Assume that $G$ contains a Carter subgroup $K$ with $S \leq K$. Since $K$ is nilpotent, it follows that $S$ is normal in $K$ and $K \leq S C_{G}(S) \unlhd N_{G}(S)$. By Feit-Thompson Theorem (see [11]) we obtain that $N_{G}(S)$ is solvable. Thus, by Lemma 2.2(1) we have that $S C_{G}(S)$ is self-normalizing in $G$, therefore $N_{G}(S)=S C_{G}(S)$.

Assume now that $N_{G}(S)=S C_{G}(S)$, i. e., $N_{G}(S)=S \times O\left(C_{G}(S)\right)$. Since $O\left(C_{G}(S)\right)$ is of odd order, it is solvable. Hence it contains a Carter subgroup $K_{1}$. Consider a nilpotent subgroup $K=S \times K_{1}$ of $G$. Assume that $x \in N_{G}(K)$, then $x \in N_{G}(S)$. But $K$ is a Carter subgroup of $N_{G}(S)$, hence $x \in K$ and $K$ is a Carter subgroup of $G$. 
Lemma 2.4. Let $G$ be a finite group, let $S$ be a Sylow 2-subgroup of $G$ and $x \in N_{G}(S)$ be of odd order. Assume that there exist normal subgroups $G_{1}, \ldots, G_{k}$ of $G$ such that $G_{1} \cap \ldots \cap$ $G_{k} \cap S \leq Z\left(N_{G}(S)\right)$. If $\varphi_{i}: G \rightarrow G_{i}$ is the natural homomorphism assume also that $x^{\varphi_{i}}$ centralizes $S G_{i} / G_{i}$.

Then $x$ centralizes $S$.

Proof. Consider the normal series $S \triangleright S_{1} \triangleright \ldots \triangleright S_{k} \triangleright S_{k+1}=\{e\}$, where $S_{i}=S \cap\left(G_{1} \cap \ldots \cap G_{i}\right)$. The conditions of the lemma implies that $x$ centralizes every factor $S_{i-1} / S_{i}$. Since $x$ is of odd order this imply that $x$ centralizes $S$.

Lemma 2.5. [9, Lemma 3] Let $G$ be a finite group. Let $H$ be a Carter subgroup of $G$. Assume that there exists a normal subgroup $B=T_{1} \times \ldots \times T_{k}$ of $G$ such that $T_{1} \simeq \ldots \simeq T_{k} \simeq T$, $Z\left(T_{i}\right)=\{1\}$ for all $i$, and $G=H\left(T_{1} \times \ldots \times T_{k}\right)$. Then $\operatorname{Aut}_{H}\left(T_{i}\right)$ is a Carter subgroup of $\left\langle\operatorname{Aut}_{H}\left(T_{i}\right), T_{i}\right\rangle$.

Lemma 2.6. Let $G$ be a finite group, let $H$ be a normal subgroup of $G$ such that $|G: H|=2^{t}$. Let $S, T$ be Sylow 2-subgroups of $G, H$ respectively and $N_{H}(T)=T C_{H}(T)$. Then $N_{G}(S)=$ $S C_{G}(S)$.

In particular, both $G, H$ contain Carter subgroups $K, L$ respectively with $S \leq K$ and $T \leq L$.

Proof. Consider $N_{G}(S)$. Since $H$ is normal in $G$ we have that

$$
N_{G}(S) \leq N_{G}(T)=\left\langle S, N_{H}(T)\right\rangle=\left\langle S, T \times O\left(N_{H}(T)\right)\right\rangle .
$$

Since $N_{H}(T)$ is normal in $N_{G}(T)$, we have that $O\left(N_{H}(S)\right)=O\left(C_{H}(T)\right)$ is normal in $N_{G}(T)$, hence $N_{G}(T)=O\left(N_{H}(T)\right) \lambda S$. Since $N_{G}(S) \leq N_{G}(T)$, we obtain that the set of elements of odd order is a normal subgroup of $N_{G}(S)$, i. e., every element of odd order of $N_{G}(S)$ is contained in $O\left(N_{G}(S)\right)$. On the other hand $S$ is normal in $N_{G}(S)$ by definition and $S \cap O\left(N_{G}(S)\right)=\{1\}$, hence $N_{G}(S)=S \times O\left(N_{G}(S)\right)=S C_{G}(S)$.

\section{Groups of Lie type}

Our notations for groups of Lie type agrees with [12] and for linear algebraic groups agrees with [13]. If $G$ is a finite group of Lie type with trivial centre (we do not exclude non-simple groups of Lie type, such as $A_{1}(2)$, all exceptions are given in [12, Theorems 11.1.2 and 14.4.1]), then $\widehat{G}$ denotes the group of inner-diagonal automorphisms of $G$. In view of $[14,3.2]$ we have that $\operatorname{Aut}(G)$ is generated by inner-diagonal, field and graph automorphisms. Since we are assuming that $Z(G)$ is trivial, we have that $G$ is isomorphic to the group of its inner automorphisms and hence we may suppose that $G \leq \widehat{G} \leq \operatorname{Aut}(G)$.

Let $\bar{G}$ be a simple connected linear algebraic group over an algebraically closed field $\mathbb{F}_{p}$ of positive characteristic $p$. It is possible here that $Z(\bar{G})$ is nontrivial. An automorphism $\sigma$ of $\bar{G}$ is called a Frobenius map if $\bar{G}_{\sigma}$ is finite. Groups $O^{p^{\prime}}\left(\bar{G}_{\sigma}\right)$ are called canonical finite groups of Lie type and every group $G$ satisfying $O^{p^{\prime}}\left(\bar{G}_{\sigma}\right) \leq G \leq \bar{G}_{\sigma}$ is called a finite group of Lie type. Note that in [12] only groups $O^{p^{\prime}}(\bar{G})$ are called groups of Lie type. But later in [15] R.Carter said that every group $\bar{G}_{\sigma}$ is a finite group of Lie type for an arbitrary connected reductive group $\bar{G}$. More over, in [16] and [17] every group $G$ with $O^{p^{\prime}}\left(\bar{G}_{\sigma}\right) \leq G \leq \bar{G}_{\sigma}$ is called a finite group of Lie type. Thus, by given definition of finite groups of Lie type and canonical finite groups of Lie type we intend to clarify the situation here. For example, $\mathbf{P} S L_{2}(3)$ is a canonical finite 
group of Lie type and $\mathbf{P} G L_{2}(3)$ is a finite group of Lie type. Note that an element of order 3 is not conjugate to its inverse in $\mathbf{P} S L_{2}(3)$ and is conjugate to its inverse in $\mathbf{P} G L_{2}(3)$. Since such information about conjugation is important in many cases (and is very important and useful in this paper), we find it reasonable to use such notation.

We say that groups ${ }^{2} A_{n}\left(q^{2}\right),{ }^{2} D_{n}\left(q^{2}\right),{ }^{2} E_{6}\left(q^{2}\right)$ are defined over $G F\left(q^{2}\right)$, groups ${ }^{3} D_{4}\left(q^{3}\right)$ are defined over $G F\left(q^{3}\right)$ and over groups are defined over $G F(q)$. The field $G F(q)$ in all cases is called the base field. In view of $[18,12.3]$ and [19, Exercise after Lemma 58] we have that if $\bar{G}$ is of adjoint type then $\bar{G}_{\sigma}$ is a group of inner-diagonal automorphisms of $O^{p^{\prime}}\left(\bar{G}_{\sigma}\right)$. If $\bar{G}$ is simply connected, then $\bar{G}_{\sigma}=O^{p^{\prime}}\left(\bar{G}_{\sigma}\right)$ (cf. $[18,12.4]$ ). In general for given finite group of Lie type $G$ (if we consider it as an abstract group) the corresponding algebraic group is not uniquely determined. For example, if $G=\mathbf{P} S L_{2}(5) \simeq S L_{2}(4)$, then $G$ can be obtained either as $\left(S L_{2}\left(\mathbb{F}_{2}\right)\right)_{\sigma}$, or as $O^{5^{\prime}}\left(\left(\mathbf{P} S L_{2}\left(\mathbb{F}_{5}\right)\right)_{\sigma}\right)$ (for appropriate $\left.\sigma\right)$. So, for any finite group of Lie type $G$, we fix (in some way) corresponding algebraic group $\bar{G}$ and a Frobenius map $\sigma$ such that $O^{p^{\prime}}\left(\bar{G}_{\sigma}\right) \leq G \leq \bar{G}_{\sigma}$. Let $U=\left\langle X_{r} \mid r \in \Phi^{+}\right\rangle$be the maximal unipotent subgroup of $G$. If we fix an order on $\Phi(\bar{G})$ consistent with the sum of roots, then every $u \in U$ can be uniquely written as

$$
u=\prod_{r \in \Phi^{+}} x_{r}\left(t_{r}\right)
$$

where roots are taken in given order and $t_{r}$ are from the field of definition of $G$. Sometimes we use notation $\Phi^{\varepsilon}(q)$, where $\varepsilon \in\{+,-\}$, and $\Phi^{+}(q)=\Phi(q)$ is a split group of Lie type with base field $G F(q), \Phi^{-}(q)={ }^{2} \Phi\left(q^{2}\right)$ is a twisted group of Lie type defined over a field $G F\left(q^{2}\right)$ (with base field $G F(q)$ ).

Now let $\bar{R}$ be a closed $\sigma$-stable subgroup of $\bar{G}$. Then we can consider $R=G \cap \bar{R}$ and $N(G, R)=G \cap N_{\bar{G}}(\bar{R})$. Note that $N(G, R) \neq N_{G}(R)$ in general and we call $N(G, R)$ an algebraic normalizer of $R$. For example, if we consider $G=S L_{n}(2)$, then the group of diagonal matrices $H$ of $G$ is trivial, hence $N_{G}(H)=G$. But $G=\left(S L_{n}\left(\mathbb{F}_{2}\right)\right)_{\sigma}$, where $\sigma$ is the Frobenius map $\sigma:\left(a_{i, j}\right) \mapsto\left(a_{i, j}^{2}\right)$. Then $H=\bar{H}_{\sigma}$, where $\bar{H}$ is the subgroup of diagonal matrices in $S L_{n}\left(\mathbb{F}_{2}\right)$. Thus $N(G, H)$ is the group of monomial matrices of $G$. We use term "algebraic normalizer" in order to avoid such difficulties, and to make our proofs universal. A group $R$ is said to be a torus (resp. a reductive subgroup, a parabolic subgroup, a maximal torus, a reductive subgroup of maximal rank) if $\bar{R}$ is a torus (resp. a reductive subgroup, a parabolic subgroup, a maximal torus, a reductive subgroup of maximal rank) of $\bar{G}$. If $\bar{R}$ is a connected reductive subgroup of maximal rank of $\bar{G}$, then $\bar{R}=\bar{G}_{1} * \ldots * \bar{G}_{k} * \bar{S}$, where $\bar{G}_{i}$ is a simple connected linear algebraic group and $\bar{S}=Z(\bar{R})^{0}$ (see [13, Theorem 27.5]). Moreover, if $\Phi_{1}, \ldots, \Phi_{k}$ are root systems of $\bar{G}_{1}, \ldots, \bar{G}_{k}$ respectively, then $\Phi_{1} \oplus \ldots \oplus \Phi_{k}$ is a subsystem of $\Phi(\bar{G})$. There is a nice algorithm due to Borel and de Siebental [20] and independently Dynkin [21] of determining subsystems of $\Phi$. One has to remove some nodes from the extended Dynkin diagram of $\Phi$. The remaining connected components are Dynkin diagrams of indecomposable components in some subsystem and any subsystem can be derived in this way.

Now assume that $\bar{R}$ is $\sigma$-stable. In view of $[18,10.10]$ there exists a $\sigma$-stable maximal torus $\bar{T}$ of $\bar{R}$. Let $\bar{G}_{1}, \ldots, \bar{G}_{l}$ be the $\sigma$-orbit of $\bar{G}_{1}$. Then

$$
\left(\bar{G}_{1} * \ldots * \bar{G}_{l}\right)_{\sigma}=\left\{x \in \bar{G}_{1} \mid x=g \cdot g^{\sigma} \cdot \ldots \cdot g^{\sigma^{l-1}} \text { for some } g \in \bar{G}\right\}_{\sigma} \simeq\left(\bar{G}_{1}\right)_{\sigma^{l}} .
$$

In view of $[18,10.15]$ we have that $\bar{G}_{\sigma^{l}}$ is finite, hence $O^{p^{\prime}}\left(\left(\bar{G}_{1}\right)_{\sigma^{l}}\right)$ is a canonical finite group of Lie type, probably, with the base field larger then the base field of $O^{p^{\prime}}\left(\bar{G}_{\sigma}\right)$. Since $\bar{G}_{1} * \ldots * \bar{G}_{l}$ is $\sigma$-stable then $\bar{G}_{1} * \ldots * \bar{G}_{l} \cap \bar{T}$ is a $\sigma$-stable maximal torus of $\bar{G}_{1} * \ldots * \bar{G}_{l}$. Therefore we may 
assume that for any $\sigma$-orbit $\left\{\bar{G}_{j_{1}}, \ldots, \bar{G}_{j_{i}}\right\} \bar{T} \cap \bar{G}_{j_{1}} * \ldots * \bar{G}_{j_{i}}$ is a maximal $\sigma$-stable torus of $\bar{G}_{j_{1}} * \ldots * \bar{G}_{j_{i}}$. For $i^{\text {th }} \sigma$-orbit $\bar{G}_{j_{1}} * \ldots * \bar{G}_{j_{i}}$ let $G_{i}=O^{p^{\prime}}\left(\bar{G}_{j_{1}} * \ldots * \bar{G}_{j_{i}}\right)_{\sigma}=O^{p^{\prime}}\left(G_{j_{1}}\right)_{\sigma^{i}}$. Then $\bar{R}_{\sigma}=\bar{T}_{\sigma}\left(G_{1} * \ldots * G_{m} * \bar{S}_{\sigma}\right)$ and $\bar{T}_{\sigma}$ normalizes each of $G_{i}$. Subgroups $G_{i}$ of $O^{p^{\prime}}\left(\bar{G}_{\sigma}\right)$ arising in this way we call subsystem subgroups of $O^{p^{\prime}}\left(\bar{G}_{\sigma}\right)$.

For a $\sigma$-orbit $\left\{\bar{G}_{j_{1}}, \ldots, \bar{G}_{j_{i}}\right\}$ of $\bar{G}_{j_{1}}$, with $G_{i}=O^{p^{\prime}}\left(\left(\bar{G}_{j_{1}}\right)_{\sigma^{s}}\right)$, consider $\operatorname{Aut}_{\bar{R}_{\sigma}}\left(G_{i}\right)$. Since $G_{1} * \ldots * G_{i-1} * G_{i+1} * \ldots * G_{k} * \bar{S}_{\sigma} \leq C_{\bar{R}_{\sigma}}\left(G_{i}\right)$, we have that $\operatorname{Aut}_{\bar{R}_{\sigma}}\left(G_{i}\right) \simeq\left(\left(\overline{T G}_{j_{1}}\right) / Z\left(\overline{T G}_{j_{1}}\right)\right)_{\sigma^{i}}$, i. e., $\operatorname{Aut}_{\bar{R}_{\sigma}}\left(G_{i}\right)$ is a finite group of Lie type and $\operatorname{Aut}_{\bar{R}_{\sigma}}\left(G_{i}\right)$ has trivial centre. Therefore we may assume that $\mathbf{P} G_{i} \leq \operatorname{Aut}_{\bar{R}_{\sigma}}\left(G_{i}\right) \leq \widehat{\mathbf{P}}_{i}$.

Let $\bar{R}$ be a $\sigma$-stable connected reductive subgroup of maximal rank (in particular, $\bar{R}$ can be a maximal torus) of $G$. Let $C l\left(\bar{G}_{\sigma}, \bar{R}\right)$ be the set of $\bar{G}_{\sigma}$-conjugacy classes of $\sigma$-stable subgroups $\bar{R}^{g}$, where $g \in \bar{G}$. Then $C l\left(\bar{G}_{\sigma}, \bar{R}\right)$ is in $1-1$ correspondence with the set of $\sigma$-conjugacy classes $C l\left(N_{W}\left(W_{\bar{R}}\right) / W_{\bar{R}}, \sigma\right)$ (we define this term below), where $W$ is the Weyl group of $\bar{G}, W_{\bar{R}}$ is the Weyl group of $\bar{R}$ (and it is a subgroup of $W$ ). Now define $C l\left(N_{W}\left(W_{\bar{R}}\right) / W_{\bar{R}}, \sigma\right)$. Since $N_{\bar{G}}(\bar{R}) / \bar{R} \simeq N_{W}\left(W_{\bar{R}}\right) / W_{\bar{R}}$ we obtain the induced action of $\sigma$ on $N_{W}\left(W_{\bar{R}}\right) / W_{\bar{R}}$ and we say that $w_{1} \equiv w_{2}$, for $w_{1}, w_{2} \in N_{W}\left(W_{\bar{R}}\right) / W_{\bar{R}}$ if there exists $w \in W$ with $w_{1}=w^{-1} w_{2} w^{\sigma}$. Now if $w$ is an element of $N_{W}\left(W_{\bar{R}}\right) / W_{\bar{R}}$, and $\left(\bar{R}^{g}\right)_{\sigma}$ corresponds to the $\sigma$-conjugated class of $w$ then we say that $\left(\bar{R}^{g}\right)_{\sigma}$ is obtained by "twisting" $\bar{R}$ with $w \sigma$. For more details see [23].

Lemma 3.1. Let $G$ be a simple connected linear algebraic group over a field of characteristic p. Let $t$ be an element of prime order $r \neq p$ of $G$.

Then $C_{G}(t) /\left(C_{G}(t)^{0}\right)$ is an r-group.

Proof. Since $r$ is distinct from the characteristic it follows that $t$ is semisimple. Hence, $C_{G}(t)^{0}$ is a connected reductive subgroup of maximal rank of $G$ and every $p$-element of $C_{G}(t)$ is contained in $C_{G}(t)^{0}$. Assume that some prime $s \neq r$ divides $\left|C_{G}(t) /\left(C_{G}(t)^{0}\right)\right|$. Then $s \neq p$ and $C_{G}(t)$ contains an element $x$ of order $s^{k}$ such that $x \notin C_{G}(t)^{0}$. Since $x, t$ commute we have that $x \cdot t$ is a semisimple element of $G$. Therefore there exists a maximal torus $T$ of $G$ with $x \cdot t \in T$. Then $(x t)^{r}=x^{r} \in T$. Since $(s, r)=1$ we have that there exists $m$ such that $r m \equiv 1\left(\bmod s^{k}\right)$, thus $\left(x^{r}\right)^{m}=x \in T$. But $T \leq C_{G}(t)^{0}$, hence $x \in C_{G}(t)^{0}$, a contradiction.

Assume now that $\bar{R}$ is a $\sigma$-stable parabolic subgroup of $\bar{G}$. Then it has the unipotent radical $\bar{U}$ and a connected reductive subgroup $\bar{L}$ such that $\bar{R} / \bar{U} \simeq \bar{L}$. The subgroup $\bar{L}$ is called a Levi factor of $\bar{R}$. Moreover, if $\bar{S}=Z(\bar{L})^{0}$, then $\bar{L}=C_{\bar{G}}(\bar{S})$. Let $\operatorname{Rad}(\bar{R})$ be the radical of $\bar{R}$. Then it is a $\sigma$-stable connected solvable subgroup, hence, by $[18,10.10]$ it contains a $\sigma$-stable torus $\bar{S}$. Now $C_{\bar{G}}(\bar{S})=C_{\bar{R}}(\bar{S})$ is a $\sigma$-stable Levi factor of $\bar{R}$, i. e., every $\sigma$-stable parabolic subgroup of $\bar{G}$ contains a $\sigma$-stable Levi factor $\bar{L}$ and $\bar{L}$ is a connected reductive subgroup of maximal rank of $\bar{G}$.

Lemma 3.2. Let $O^{p^{\prime}}\left(\bar{G}_{\sigma}\right) \leq G \leq \bar{G}_{\sigma}$ be a finite group of Lie type over a field of odd characteristic $p$ and the root system $\Phi$ of $\bar{G}$ be one of the following: $A_{\ell}(\ell \geqslant 2), D_{\ell}(\ell \geqslant 3), B_{\ell}(\ell \geqslant 3), E_{6}$, $E_{7}$ or $E_{8}$ and $G \neq^{3} D_{4}\left(q^{3}\right)$. Let $U$ be a maximal unipotent subgroup of $G$ and let $H$ be a Cartan subgroup of $G$ which normalizes $U$. Then $C_{U}(\Omega(H))=\{1\}$, where $\Omega(H)=\left\{h \in H \mid h^{2}=1\right\}$.

Proof. For split case and twisted case with $\Phi=D_{\ell}$, the lemma is proven in [10, Lemma 2.8]. The remaining cases can be proven by using the same arguments.

Lemma 3.3. Let $O^{p^{\prime}}\left(\bar{G}_{\sigma}\right)=G$ be a canonical finite group of Lie type over a field of odd characteristic $p$ and -1 is not a square in the base field of $G$. Assume that the root system $\Phi$ of $\bar{G}$ is equal to $C_{\ell}$. Let $U$ be a maximal unipotent subgroup of $G$ and let $H$ be a Cartan subgroup 
of $G$ which normalizes $U$. Then $C_{U}(\Omega(H))=\left\langle X_{r}\right| r$ is a long root $\rangle$, where $\Omega(H)=\{h \in H \mid$ $\left.h^{2}=1\right\}$.

Proof. If $r$ is a short root, then there exists a root $s$ with $<s, r>=1$. Thus $x_{r}(t)^{h_{s}(-1)}=$ $x_{r}\left((-1)^{<s, r>} t\right)=x_{r}(-t)$ (cf. [12, Proposition 6.4.1]). Therefore, if $x \in C_{U}\left(\Omega(H)\right.$ ) and $x_{r}(t)$ is a nontrivial multiplier in decomposition (1) of $x$, then $r$ is a long root. Now if $r$ is a long root, then, for every root $s$, either $|\langle s, r\rangle|=2$, or $\langle s, r\rangle=0$, i. e., $x_{r}(t)^{h_{s}(-1)}=x_{r}(t)$. Under our conditions $\left\langle h_{s}(-1) \mid s \in \Phi\right\rangle=\Omega(H)$, and the lemma follows.

The following lemma is immediate from [24, Theorem 1].

Lemma 3.4. Let $O^{p^{\prime}}\left(\bar{G}_{\sigma}\right)=G$ be a canonical finite group of Lie type and $\bar{G}$ is either of type $A_{n}$ or of type $C_{n}, p$ is odd, $q=p^{\alpha}$ is the order of the base field of $G$, and $G$ is split. Let $S$ be a Sylow 2-subgroup of $G$.

Then $N_{G}(S)=S C_{G}(S)$ if and only if $q \equiv \pm 1(\bmod 8)$.

Lemma 3.5. Let $O^{p^{\prime}}\left(\bar{G}_{\sigma}\right) \leq G \leq \bar{G}_{\sigma}$ be a finite group of Lie type with the base field of characteristic $p$ and order $q$, let $\bar{G}$ be of adjoint type. Assume also that $G$ is not isomorphic to ${ }^{2} D_{2 n}\left(q^{2}\right),{ }^{3} D_{4}\left(q^{3}\right),{ }^{2} B_{2}\left(2^{2 n+1}\right),{ }^{2} G_{2}\left(3^{2 n+1}\right),{ }^{2} F_{4}\left(2^{2 n+1}\right)$. Then there exists a maximal $\sigma$-stable torus $\bar{T}$ of $\bar{G}$ such that

(1) $\left(N_{\bar{G}}(\bar{T}) / \bar{T}\right)_{\sigma} \simeq\left(N_{\bar{G}}(\bar{T})\right)_{\sigma} /\left(\bar{T}_{\sigma}\right)=N\left(\bar{G}_{\sigma}, \bar{T}_{\sigma}\right) / \bar{T}_{\sigma} \simeq W$, where $W$ is the Weyl group of $\bar{G}$;

(2) if $r$ is a prime divisor of $q-(\varepsilon 1)$, where $\varepsilon=+$, if $G$ is split and $\varepsilon=-$ if $G$ is twisted, then, up to conjugation in $O^{p^{\prime}}\left(\bar{G}_{\sigma}\right)$, every element of order $r$ is contained in $\bar{T}_{\sigma}$;

(3) torus $\bar{T}$ is unique, up to conjugation in $O^{p^{\prime}}\left(\bar{G}_{\sigma}\right)$.

Proof. Since for every maximal torus $T$ of $\bar{G}_{\sigma}$ we have that $\bar{G}_{\sigma}=T O^{p^{\prime}}\left(\bar{G}_{\sigma}\right)$, without lost we may assume that $G=\bar{G}_{\sigma}$. If $G$ is split then the lemma is evident. In this case $\bar{T}$ is a maximal torus such that $\bar{T}_{\sigma}$ is a Cartan subgroup of $\bar{G}_{\sigma}$ and (1) is clear. By $[25, \mathrm{~F}, \S 6]$ we have that every element of order $r$ of $\bar{G}_{\sigma}$, up to conjugation, is contained in $\bar{T}_{\sigma}$ and (2) follows. By information about the classes of maximal tori given in $[25, \mathrm{G}]$ and $[26]$ we have that $\bar{T}$ is unique, up to conjugation in $G$.

Assume that $G \simeq{ }^{2} A_{n}\left(q^{2}\right)$. Then $\bar{T}$ is a maximal torus such that $\left|\bar{T}_{\sigma}\right|=(q+1)^{n}$. The uniqueness follows from [16, Proposition 8]. Direct calculations by using [15, Proposition 3.3.6] show that $N\left(\bar{G}_{\sigma}, \bar{T}_{\sigma}\right) / \bar{T}_{\sigma} \simeq W(\bar{G})=$ Sym $_{n+1}$. Assume that $t$ is an element of order $r$ in $G$ (recall that in this case $r$ divides $q+1$ ). Let $S=A_{n}\left(q^{2}\right)$ be chosen so that $G=S_{\tau}$ for some automorphism $\tau$ (the existence of such group follows from [12, Chapter 13]). Moreover there exists a Frobenius map $\rho$ of $\bar{G}$ such that $S=\bar{G}_{\rho}$ and $\rho=\sigma^{2}$. Now let $\bar{H}$ be a $\sigma$-stable maximal torus of $\bar{G}$ such that $\bar{H}_{\sigma}$ is a Cartan subgroup of $G$. Then $\bar{H}$ is also $\rho$-stable and $\bar{H}_{\rho}$ is a Cartan subgroup of $S$. In view of $[25, \mathrm{~F}, \S 6]$ we have that $t$ is contained in $\bar{H}_{\rho}$. We state that, up to conjugation in $S$, the torus $\bar{T}_{\sigma}$ is contained in $\bar{H}_{\rho}$. Indeed, $\bar{T}_{\sigma}$ is obtained from $\bar{H}$ by "twisting" with $w_{0} \sigma$, where $w_{0} \in W(\bar{G})$ is the unique element that maps all positive roots onto negative roots. Now $\bar{T}_{\rho}$ is obtained from $\bar{H}$ by "twisting" with an element $\left(w_{0} \sigma\right)^{2}=w_{0}^{2} \rho=\rho$, i. e., $\bar{T}_{\rho}$ and $\bar{H}_{\rho}$ are conjugate in $S$. Let $r_{1}, \ldots, r_{n}$ be the set of fundamental roots of $A_{n}$. Then $t$, as an element of $\bar{H}_{\rho}$ can be written as $h_{r_{1}}\left(\zeta_{1}\right) \cdot \ldots \cdot h_{r_{n}}\left(\zeta_{n}\right)$. Now $\bar{T}_{\sigma}=\left(\bar{H}_{\rho}\right)_{\tau w_{0}}$. But $\tau w_{0}: h_{r}(\lambda) \mapsto h_{-r}\left(\lambda^{q}\right)=h_{r}\left(\lambda^{-q}\right)$, i. e. $t^{\tau w_{0}}=t^{-q}$. Now assume that $t$ is of order $r$. Since $r$ divides $q+1$ we obtain that $t^{q+1}=e$, i. e., $t=t^{-q}$. Hence $t^{\tau w_{0}}=t$ and $t \in \bar{T}_{\sigma}$. 
For $G={ }^{2} D_{2 n+1}\left(q^{2}\right)$ we take $\bar{T}$ to be the unique (up to conjugation in $G$ ) maximal torus of order $\left|\bar{T}_{\sigma}\right|=(q+1)^{2 n+1}$ (the uniqueness follows from [16, Proposition 10]) and for $G={ }^{2} E_{6}\left(q^{2}\right)$ we take $\bar{T}$ to be the unique (again up to conjugation in $G$ ) maximal torus of order $\left|\bar{T}_{\sigma}\right|=(q+1)^{6}$ (the uniqueness follows from [17, Table 1, p. 128]). Like in case $G={ }^{2} A_{n}\left(q^{2}\right)$ it is easy to show that $\bar{T}$ satisfies (1) and (2) of the lemma.

Lemma 3.6. Let $G$ be a finite group of Lie type and $\bar{G}$, $\sigma$ are chosen so that $O^{p^{\prime}}\left(\bar{G}_{\sigma}\right) \leq G \leq \bar{G}_{\sigma}$. Let $s$ be a regular semisimple element of odd prime order of $G$.

Then $N_{G}\left(C_{G}(s)\right) \neq C_{G}(s)$.

Proof. In view of $\left[25, \mathrm{~F}, \S 4\right.$ and Proposition 5] we have that $C_{\bar{G}}(s) / C_{\bar{G}}(s)^{0}$ is isomorphic to a subgroup of $\Delta$. Now, if the root system $\Phi$ of $\bar{G}$ is not equal to either $A_{n}$, or $E_{6}$, then $|\Delta|$ is a power of 2. Thus, Lemma 3.1 implies that $C_{\bar{G}}(s)=C_{\bar{G}}(s)^{0}=\bar{T}$ and $C_{G}(s)=C_{\bar{G}}(s) \cap G=T$. Since $N_{G}(T) \geq N(G, T) \neq T$ we obtain the statement in this case. Thus we may assume that either $\Phi=A_{n}$, or $\Phi=E_{6}$.

Assume first that $\Phi=A_{n}$, i. e., $G=A_{n}^{\varepsilon}(q)$, where $\varepsilon \in\{+,-\}$. Clearly $T=C_{\bar{G}}(s)^{0} \cap G$ is a normal subgroup of $C_{G}(s)$, hence $C_{G}(s) \leq N(G, T)$. Assume that $N_{G}\left(C_{G}(s)\right)=C_{G}(s)$. Then $C_{G}(s)=N_{N(G, T)}\left(C_{G}(s)\right)$ and $C_{G}(s) / T$ is a self-normalizing subgroup of $N(G, T) / T$. As we noted above $C_{G}(s) / T$ is isomorphic to a subgroup of $\Delta$, i. e., it is cyclic. By Lemma 3.1, we also have that $C_{G}(s) / T$ is an $r$-group, thus $C_{G}(s) / T=\langle x\rangle$ for some $x \in N(G, T) / T$ and $\langle x\rangle$ is a Carter subgroup of $N(G, T) / T$. Now, in view of [15, Proposition 3.3.6], we have that $N(G, T) / T \simeq C_{\operatorname{Sym}_{n+1}}(y)$ for some $y \in \operatorname{Sym}_{n+1}$. Clearly $C_{C_{\operatorname{Sym}_{n+1}}(y)}(x)$ contains $y$, thus $y$ must be an $r$-element, otherwise $N_{C_{\mathrm{Sym}_{n+1}}(y)}(\langle x\rangle)$ would contain an element of order coprime to $r$, i. e., $N_{C_{\mathrm{Sym}_{n+1}}(y)}(\langle x\rangle) \neq\langle x\rangle$. A contradiction with the fact that $\langle x\rangle$ is a Carter subgroup of $C_{\mathrm{Sym}_{n+1}}(y)$.

Now let $y=\tau_{1} \cdot \ldots \cdot \tau_{k}$ be the decomposition of $y$ into the product of independent cycles and $l_{1}, \ldots, l_{k}$ be the lengths of $\tau_{1}, \ldots, \tau_{k}$ respectively. Assume that first $m_{1}$ cycles has the same length $l_{1}, m_{2}$ cycles has the length $l_{2}$ etc. Let $m_{0}=n+1-\left(l_{1}+\ldots+l_{k}\right)$. Then

$$
C_{\operatorname{Sym}_{n+1}}(y) \simeq\left(\left(Z_{l_{1}} \times \ldots \times Z_{l_{k}}\right) 入\left(\operatorname{Sym}_{m_{1}} \times \operatorname{Sym}_{m_{2}} \times \ldots\right)\right) \times \operatorname{Sym}_{m_{0}}
$$

where $Z_{l_{i}}$ is a cyclic group of order $l_{i}$. If $m_{j}>1$ for some $j \geqslant 0$, then there exists a normal subgroup $H$ of $C_{\mathrm{Sym}_{n+1}}(y)$ such that $C_{\mathrm{Sym}_{n+1}}(y) / H \simeq \operatorname{Sym}_{m_{j}} \neq\{e\}$. In view of [9, Table] and $[10$, Table $]$ we obtain that Carter subgroup in group $S$ satisfying $\mathrm{Alt}_{\ell} \leq S \leq \mathrm{Sym}_{\ell}$ are conjugate for all $\ell \geqslant 1$. Thus $C_{\mathrm{Sym}_{n+1}}(y)$ and $H$ satisfy $(*)$ and $\langle x\rangle$ is the unique, up to conjugation, Carter subgroup of $C_{\operatorname{Sym}_{n+1}}(y)$. By Lemma 2.1 we obtain that $\langle x\rangle$ maps onto a Carter subgroup of $C_{\mathrm{Sym}_{n+1}}(y) / H \simeq \mathrm{Sym}_{m_{j}}$. In view of [2] we have that only a Sylow 2-subgroup of $\mathrm{Sym}_{m_{j}}$ can be a Carter subgroup of $\operatorname{Sym}_{m_{j}}$. A contradiction with the fact that $x$ is an $r$-element and $r$ is odd.

Thus we may assume that $C_{\operatorname{Sym}_{n+1}}(y)=\left(Z_{l_{1}} \times \ldots \times Z_{l_{k}}\right)$ and $l_{i} \neq l_{j}$ if $i \neq j$. From the known structure of maximal tori and their normalizers of $A_{n}^{\varepsilon}(q)$ (cf. [16, Propositions 7,8], for example) we obtain that $T=\left(\left(T_{1} \times \ldots \times T_{k}\right) / Z\right) \cap A_{n}^{\varepsilon}(q)$, where $T_{i}$ is a, so-called, Singer group of $G L_{l_{i}}^{\varepsilon}(q)=G_{i}$ and $N(G, T)=\left(\left(N\left(G_{1}, T_{1}\right) \times \ldots \times N\left(G_{k}, T_{k}\right)\right) / Z\right) \cap A_{n}^{\varepsilon}(q)$. Thus we may assume that $N(G, T)=C_{G}(s)$ and $T$ is a Singer group, i. e., it is a cyclic group of order $\frac{q^{n+1}-(\varepsilon 1)^{n+1}}{q-(\varepsilon 1)}$. It is known that if $x$ generates $N(G, T)$ modulo $T$, then $T_{x}$ is in the centre of $G$, therefore, $x \notin C_{G}(s)$ (for details see [27, p. 187]).

In the remaining case $\Phi=E_{6}$ by direct calculation, using [33], for example, it is easy to check that, for every $y \in W\left(E_{6}\right), C_{W\left(E_{6}\right)}(y)$ does not contain Carter subgroups of order 3 . Since 
$\left|C_{G}(s) / T\right|$ divides 3 and $C_{G}(s) / T$ is a Carter subgroup in $C_{W\left(E_{6}\right)}(y)$ for some $y$, we obtain a contradiction.

\section{Semilinear groups of Lie type}

Now we define some special overgroups of finite groups of Lie type. First we give precise description of a Frobenius map $\sigma$. Let $\bar{G}$ be a simple connected linear algebraic group over an algebraically closed field $\mathbb{F}_{p}$ of positive characteristic $p$. Choose a Borel subgroup $\bar{B}$ of $\bar{G}$, let $\bar{U}=R_{u}(\bar{B})$ be the unipotent radical of $\bar{B}$. There exists a Borel subgroup $\bar{B}^{-}$with $\bar{B} \cap \bar{B}^{-}=\bar{T}$, where $\bar{T}$ is a maximal torus of $\bar{B}$ (hence of $\bar{G}$ ). Let $\Phi$ be the root system of $\bar{G}$ and let $\left\{X_{r} \mid r \in \Phi^{+}\right\}$be the set of $\bar{T}$-invariant 1-dimensional root subgroups of $\bar{U}$. Every $X_{r}$ is isomorphic to the additive group of $\mathbb{F}_{p}$, so every element of $X_{r}$ can be written as $x_{r}(t)$, where $t$ is the image of $x_{r}(t)$ under above mentioned isomorphism. Denote by $\bar{U}^{-}=R_{u}\left(\bar{B}^{-}\right)$the unipotent radical of $\bar{B}^{-}$. As above define $\bar{T}$-invariant 1-dimensional subgroups $\left\{\bar{X}_{r} \mid r \in \Phi^{-}\right\}$of $\bar{U}^{-}$. Then $\bar{G}=\left\langle\bar{U}, \bar{U}^{-}\right\rangle$. Let $\bar{\varphi}$ be a field automorphism of $\bar{G}$ and $\bar{\gamma}$ be a graph automorphism of $\bar{G}$. It is known that $\bar{\varphi}$ can be chosen so that it acts by $x_{r}(t)^{\bar{\varphi}}=x_{r}\left(t^{p}\right)$ (see $[12,12.2]$ and $[15$, 1.7], for example). In view of [12, Proposition 12.2.3 and Proposition 12.3.3] we can choose $\bar{\gamma}$ so that it acts by $x_{r}(t)^{\bar{\gamma}}=x_{\bar{r}}(t)$ if $\Phi$ has no roots of distinct length or by $x_{r}(t)^{\bar{\gamma}}=x_{\bar{r}}\left(t^{\lambda}\right)$ for appropriate $\lambda$ if $\Phi$ has roots of distinct length. Here $\bar{r}$ is the image of $r$ under the symmetry $\rho$, corresponding to $\bar{\gamma}$, of root system $\Phi$. In both cases we can write $x_{r}(t)^{\bar{\gamma}}=x_{\bar{r}}\left(t^{\lambda}\right)$, where $\lambda$ is a field automorphism (probably trivial) of $\mathbb{F}_{p}$. From these formulae it is evident that $\bar{\varphi} \cdot \bar{\gamma}=\bar{\gamma} \cdot \bar{\varphi}$. Let $n_{r}(t)=x_{r}(t) x_{-r}\left(-t^{-1}\right) x_{r}(t)$ and $\bar{N}=\left\langle n_{r}(t) \mid r \in \Phi, t \in \mathbb{F}_{p}\right\rangle$. Let $h_{r}(t)=n_{r}(t) n_{r}(-1)$ and $\bar{H}=\left\langle h_{r}(t) \mid r \in \Phi, t \in \mathbb{F}\right\rangle$. In view of [12, Chapters 6 and 7$], \bar{H}$ is a maximal torus of $\bar{G}$, $\bar{N}=N_{\bar{G}}(\bar{H})$ and $\bar{X}_{r}$ are root subgroups with respect to $\bar{H}$. So we can substitute $\bar{T}$ by $\bar{H}$ and suppose that under our choice $\bar{T}$ is $\bar{\varphi}$ - and $\bar{\gamma}$ - invariant. Moreover $\bar{\varphi}$ induces the trivial automorphism of $\bar{N} / \bar{T}$.

Automorphism $\bar{\varphi}^{k}, k \in \mathbb{N}$ is called a classical Frobenius automorphism. We shall call an automorphism $\sigma$ a Frobenius automorphism if $\sigma$ is conjugate under $\bar{G}$ to $\bar{\gamma}^{e} \bar{\varphi}^{k}, e \in\{0,1\}, k \in \mathbb{N}$. It follows from Lang-Steinberg theorem [18, Theorem 10.1] that for any $\bar{g} \in \bar{G}$, elements $\sigma$ and $\sigma \bar{g}$ are conjugate under $\bar{G}$. Thus, in view of $[18,11.6]$, we have that a Frobenius map, defined in previous section, coincides with a Frobenius automorphism defined here.

Now fix $\bar{G}, \bar{\varphi}, \bar{\gamma}$, and $\sigma=\bar{\gamma}^{e} \bar{\varphi}^{k}$; and assume that $|\bar{\gamma}| \leqslant 2$, i. e., we do not consider the triality automorphism of $D_{4}$. Consider $B=\bar{B}_{\sigma}, T=\bar{T}_{\sigma}$, and $U=\bar{U}_{\sigma}$. Since $\bar{B}, \bar{T}$, and $\bar{U}$ are $\bar{\varphi}$ - and $\bar{\gamma}$ - invariant, they give us Borel subgroup, Cartan subgroup, and maximal unipotent subgroup (Sylow $p$-subgroup) of $\bar{G}_{\sigma}$ (see $[15,1.7-1.9]$ for details).

Assume first that $e=0$, i. e., $O^{p^{\prime}}\left(\bar{G}_{\sigma}\right)$ is not twisted (is split). Then $U=\left\langle X_{r} \mid r \in \Phi^{+}\right\rangle$, where $X_{r}$ is isomorphic to the additive group of $G F\left(p^{k}\right)=G F(q)$ and every element of $X_{r}$ can be written in the form $x_{r}(t), t \in G F(q)$. Consider also $U^{-}=\bar{U}_{\sigma}^{-}$. As for $U$ we can write $U^{-}=\left\langle X_{r} \mid r \in \Phi^{-}\right\rangle$and every element of $X_{r}$ can be written in the form $x_{r}(t), t \in G F(q)$. Now we can define an automorphism $\varphi$ by the restriction of $\bar{\varphi}$ on $\bar{G}_{\sigma}$ and automorphism $\gamma$ by the restriction of $\bar{\gamma}$ on $\bar{G}_{\sigma}$. By definition we have that $x_{r}(t)^{\varphi}=x_{r}\left(t^{p}\right)$ and $x_{r}(t)^{\gamma}=x_{\bar{r}}\left(t^{\lambda}\right.$ ) (see the definition of $\bar{\gamma}$ above) for all $r \in \Phi$. Define $\zeta=\gamma^{\varepsilon} \varphi^{\ell}, \varphi^{\ell} \neq e, \varepsilon \in\{0,1\}$ to be an automorphism of $\bar{G}_{\sigma}$ and define $\bar{\zeta}=\bar{\gamma}^{\varepsilon} \cdot \bar{\varphi}^{\ell}$ to be an automorphism of $\bar{G}$. Choose a $\zeta$-invariant subgroup $G$ with $O^{p^{\prime}}\left(\bar{G}_{\sigma}\right) \leq G \leq \bar{G}_{\sigma}$. Note that if the root system $\Phi$ of $\bar{G}$ is not $D_{2 \ell}$, then $\bar{G}_{\sigma} /\left(O^{p^{\prime}}\left(\bar{G}_{\sigma}\right)\right)$ is cyclic. Thus for most groups and automorphisms, except groups of type $D_{2 \ell}$ over a field of 
odd characteristic, any subgroup $G$ of $\bar{G}_{\sigma}$ satisfying $O^{p^{\prime}}\left(\bar{G}_{\sigma}\right) \leq G \leq \bar{G}_{\sigma}$ is $\gamma$ - and $\varphi$ - invariant. Denote $\Gamma G=G \lambda\langle\zeta\rangle$ and $\Gamma \bar{G}=\bar{G} \lambda\langle\bar{\zeta}\rangle$.

Assume now that $e=1$, i. e., $O^{p^{\prime}}\left(\bar{G}_{\sigma}\right)$ is twisted. Then $U=\bar{U}_{\sigma}$ is generated by groups $X_{R}$, where

$$
\left.X_{R}=\left\langle\bar{X}_{r}\right| r \in\left\{\alpha s+\beta s^{\rho} \mid \alpha, \beta \geqslant 0, \alpha, \beta \in \mathbb{Z}\right\} \cap \Phi^{+} \text {for some } s \in \Phi^{+}\right\rangle_{\sigma}
$$

and $\rho$ is the symmetry of Dynkin diagram corresponding to $\bar{\gamma}, U^{-}=\bar{U}^{-}$is generated by groups

$$
\left.X_{R}=\left\langle\bar{X}_{r}\right| r \in\left\{\alpha s+\beta s^{\rho} \mid \alpha, \beta \geqslant 0, \alpha, \beta \in \mathbb{Z}\right\} \cap \Phi^{-} \text {for some } s \in \Phi^{-}\right\rangle_{\sigma} .
$$

Define $\varphi$ on $U^{ \pm}$to be the restriction of $\bar{\varphi}$ on $U^{ \pm}$. Since $O^{p^{\prime}}\left(\bar{G}_{\sigma}\right)=\left\langle U^{+}, U^{-}\right\rangle$we obtain the automorphism $\varphi$ of $O^{p^{\prime}}\left(\bar{G}_{\sigma}\right)$. Consider $\zeta=\varphi^{\ell} \neq e$ and let $G$ be a $\zeta$-invariant group with $O^{p^{\prime}}\left(\bar{G}_{\sigma}\right) \leq G \leq \bar{G}_{\sigma}$. Then $\bar{\zeta}=\bar{\varphi}^{\ell}$ is an automorphism of $\bar{G}$. Define $\Gamma G=G \lambda\langle\zeta\rangle$ and $\Gamma \bar{G} \lambda\langle\bar{\zeta}\rangle$.

A group $\Gamma G$ defined above is called a semilinear finite group of Lie type (it is called a semilinear canonical finite group of Lie type if $\left.G=O^{p^{\prime}}\left(\bar{G}_{\sigma}\right)\right)$ and $\Gamma \bar{G}$ is called a semilinear algebraic group. Note that $\Gamma \bar{G}$ can not be defined without $\Gamma G$, since we need to know that $\varphi^{\ell} \neq e$. If $G$ is written in notations of [12], i. e. $G=A_{n}(q)$ or $G={ }^{2} A_{n}\left(q^{2}\right)$ etc., then we shall write $\Gamma G$ by $\Gamma A_{n}(q), \Gamma^{2} A_{n}\left(q^{2}\right)$, etc.

Consider $x \in \Gamma G \backslash G$. Then $x=\zeta^{k} g$ for some $k \in \mathbb{N}$ and $g \in G$. Define $\bar{x}$ to be $\bar{\zeta}^{k} g$. Conversely, if $\bar{x}=\bar{\zeta}^{k} g$ for some $g \in G$ and $\zeta^{k} \neq e$, define $x$ to be equal to $\zeta^{k} g$. Note that we do not need to suppose that $\bar{x} \in \Gamma \bar{G} \backslash \bar{G}$ since $|\bar{\zeta}|=\infty$. If $x \in G$ we define $\bar{x}=x$.

Lemma 4.1. Let $H$ be a subgroup of $G$. Then $x$ normalizes $H$ if and only if $\bar{x}$ normalizes $H$ as a subgroup of $\bar{G}$.

Proof. Since $\zeta$ is the restriction of $\bar{\zeta}$ on $G$ our statement is trivial.

Let $H_{1}$ be a subgroup of $\Gamma G$. Then $H_{1}$ is generated by $H=H_{1} \cap G$ and an element $x=\zeta^{k} g$, moreover $H$ is a normal subgroup of $H_{1}$. In view of Lemma 4.1 we can consider $\bar{H}_{1}=\langle\bar{x}, H\rangle$. Now we find it reasonable to explain, why we use such complicate notations and definitions. We have that $\zeta$ is always of finite order, but $\bar{\zeta}$ is always of infinite order. Thus, even if $Z(G)$ is trivial, we can not consider $G \lambda\langle\bar{\zeta}\rangle$ as a subgroup of Aut $(G)$. Hence, we need to define in some way (one possible way is just given) the connection between elements of $\operatorname{Aut}(G)$ and elements of $\operatorname{Aut}(\bar{G})$ in order to use the machinery of linear algebraic groups.

Let $\bar{R}$ be a $\sigma$-stable maximal torus (resp. reductive subgroup of maximal rank, parabolic subgroup) of $\bar{G}$, let $y \in N_{\Gamma \bar{G}}(\bar{R})$ be chosen so that there exists $x \in \Gamma G$ with $y=\bar{x}$. Then $R_{1}=\langle x, \bar{R} \cap G\rangle$ is called a maximal torus (resp. a reductive subgroup of maximal rank, a parabolic subgroup) of $\Gamma G$.

Lemma 4.2. Let $M=\langle x, X\rangle$ be a subgroup of $\Gamma G$ such that $X=M \cap G \unlhd M$ and $O_{p}(X)$ is nontrivial. Then there exists $\sigma$-and $\bar{x}$ - stable parabolic subgroup $\bar{P}$ of $\bar{G}$ such that $X \leq \bar{P}$ and $O_{p}(X) \leq R_{u}(\bar{P})$.

Proof. Define $U_{0}=O_{p}(X), N_{0}=N_{\bar{G}}\left(U_{0}\right)$. Then $U_{i}=R_{u}\left(N_{i-1}\right)$ and $N_{i}=N_{\bar{G}}\left(U_{i}\right)$. Clearly $U_{i}, N_{i}$ are $\bar{x}_{\text {- }}$ and $\sigma$ - stable for all $i$. In view of [13, Proposition 30.3], the chain of subgroups $N_{0} \leq N_{1} \leq \ldots \leq N_{k} \leq \ldots$ is finite and $\bar{P}=\cup_{i} N_{i}$ is a proper parabolic subgroup. Clearly $\bar{P}$ is $\sigma$ - and $\bar{x}$ - stable.

Lemma 4.3. Let $G$ be a finite group of Lie type over a field of odd characteristic $p$ and $G \not$ ${ }^{2} G_{2}\left(3^{2 n+1}\right),{ }^{3} D_{4}\left(q^{3}\right),{ }^{2} D_{2 n}\left(q^{2}\right)$. Assume that $\bar{G}$ and $\sigma$ are chosen so that $O^{p^{\prime}}\left(\bar{G}_{\sigma}\right) \leq G \leq \bar{G}_{\sigma}$. Let $\psi$ be a field automorphism of $O^{p^{\prime}}\left(\bar{G}_{\sigma}\right)$ of odd order. 
Then a Sylow 2-subgroup of $G_{\psi}$ is a Sylow 2-subgroup of $G$. Moreover there exists a maximal torus $T$ of $G$ such that $N(G, T) / T \simeq N_{\bar{G}}(\bar{T}) / \bar{T}$, a Sylow 2-subgroup of $T_{\psi}$ is a Sylow 2-subgroup of $T$, and $\bar{\psi}$ normalizes every $\bar{T}$-invariant root subgroup $\bar{X}$ of $\bar{G}$.

Proof. Assume that $|\psi|=k$. Let $G F(q)$ be the base field of $G$. Then $q=p^{\alpha}$ and $\alpha=k \cdot m$. It is easy to check that every field automorphism of odd order centralizes the Sylow 2-subgroup of $\bar{G}_{\sigma} /\left(O^{p^{\prime}}\left(\bar{G}_{\sigma}\right)\right)$, hence we may assume that $G=\bar{G}_{\sigma}$. Now $|G|$ can be written as $|G|=q^{N}\left(q^{m_{1}}+\right.$ $\left.\varepsilon_{1} 1\right) \cdot \ldots \cdot\left(q^{m_{n}}+\varepsilon_{n} 1\right)$ for some $N$, where $n$ is the rank of $G, \varepsilon_{i}= \pm$. (cf. [12, Theorems 9.4.10 and 14.3.1]). Similarly we have that $\left|G_{\psi}\right|=\left(p^{m}\right)^{N}\left(\left(p^{m}\right)^{m_{1}}+\varepsilon_{1} 1\right) \cdot \ldots \cdot\left(\left(p^{m}\right)^{m_{n}}+\varepsilon_{n} 1\right)$, i. e., $|G|_{2}=\left|G_{\psi}\right|_{2}$ and a Sylow 2-subgroup of $G_{\psi}$ is a Sylow 2-subgroup of $G$.

Now, by Lemma 3.5, there exists a maximal torus $T$ of $G_{\psi}$ such that $N\left(G_{\psi}, T\right) / T \simeq$ $N_{\bar{G}}(\bar{T}) / \bar{T}$ and $\left|T_{\psi}\right|=\left(p^{m}-\varepsilon 1\right)^{n}$. Clearly $|\bar{T} \cap G|=(q-\varepsilon 1)^{n}$. If $G$ is split, then $T_{\psi}$ is a Cartan subgroup of $G_{\psi}$ and the lemma is evident. Thus we may assume that $G$ and $G_{\psi}$ are twisted, in particular, $\varepsilon=-$. In view of proof of Lemma 3.5, we have that there exists a split group $L$ such that $G_{\psi}=L_{\lambda}$ for some automorphism $\lambda$ of order $2, \bar{T} \cap L$ is a Cartan subgroup of $L$, and $\psi$ can be considered as a field automorphism of $L$. Therefore for every $\bar{T}$-root subgroup $\bar{X}$ of $\bar{G}, \bar{X} \cap L$ is a root subgroup of $L$, and it is $\psi$-invariant. Hence $\bar{X}$ is $\bar{\psi}$-invariant.

Lemma 4.4. [29, (7-2)] Let $\bar{G}$ be a connected simple linear algebraic group over a field of characteristic $p, \sigma$ be a Frobenius map of $\bar{G}$ and $G=\bar{G}_{\sigma}$ be a finite group of Lie type. Let $\varphi$ be a field or a graph-field automorphism of $G$ and let $\varphi^{\prime}$ be an element of $\left.(G\rangle\langle\varphi\rangle\right) \backslash G$ such that $\left|\varphi^{\prime}\right|=|\varphi|$.

Then there exists an element $g \in G$ such that $\langle\varphi\rangle^{g}=\left\langle\varphi^{\prime}\right\rangle$. In particular, if $G / O^{p^{\prime}}(G)$ is a 2 -group and $\varphi$ is of odd order, then such $g$ can be chosen in $O^{p^{\prime}}(G)$.

The following lemma is proven for classical groups in [28].

Lemma 4.5. Let $G$ be a finite group of Lie type with $Z(G)=\{1\}, \bar{G}, \sigma$ are chosen so that $O^{p^{\prime}}\left(\bar{G}_{\sigma}\right) \leq G \leq \bar{G}_{\sigma}$ and $Z(\bar{G})=\{1\}$. Assume that $\tau$ is the graph automorphism of order 2 .

Then every semisimple element $s \in G$ is conjugate to its inverse under $\langle\tau\rangle \wedge\left(O^{p^{\prime}}\left(\bar{G}_{\sigma}\right)\right)$.

Proof. In view of $[14,3.2]$ we have that if $G$ has a graph automorphism of order 2 , then $G$ is split. If $\bar{G}$ is not of type $A_{n}, D_{2 n+1}, E_{6}$, then the lemma follows from [10, Lemma 2.2], thus we need to consider groups of type $A_{n}, D_{2 n+1}, E_{6}$. Denote by $\bar{\tau}$ the graph automorphism of $\bar{G}$ such that $\left.\bar{\tau}\right|_{G}=\tau$. Let $\bar{T}$ be a maximal $\sigma$-stable torus of $\bar{G}$ such that $\bar{T}_{\sigma} \cap G$ is a Cartan subgroup of $G$. Let $r_{1}, \ldots, r_{n}$ be fundamental roots of $\Phi(\bar{G})$ and $\rho$ be the symmetry corresponding to $\bar{\tau}$. Denote $r_{i}^{\rho}$ by $\bar{r}_{i}$. Then $\bar{T}=\left\langle h_{r_{i}}\left(t_{i}\right)\right|$, where $1 \leqslant i \leqslant n$ and $\left.t_{i} \neq 0\right\rangle$ and $h_{r_{i}}\left(t_{i}\right)^{\bar{\tau}}=h_{\bar{r}_{i}}\left(t_{i}\right)$. Denote by $W$ the Weyl group of $\bar{G}$. Let $w_{0}$ be the unique element of $W$ mapping all positive roots onto negative roots and let $n_{0}$ be its preimage in $N_{\bar{G}}(\bar{T})$ under the natural homomorphism $N_{\bar{G}}(\bar{T}) \rightarrow N_{\bar{G}}(\bar{T}) / \bar{T} \simeq W$. Since $\sigma$ acts trivially on $W=N(G, T) / T$, we can take $n_{0} \in G$, i. e., $n_{0}^{\sigma}=n_{0}$. Then for all $r_{i}$ and $t$ we have that

$$
h_{r_{i}}(t)^{n_{0} \bar{\tau}}=h_{r_{i}^{w_{0} \rho}}(t)=h_{-r_{i}}(t)=h_{r_{i}}\left(t^{-1}\right)
$$

Thus $x^{n_{0} \bar{\tau}}=x^{-1}$ for all $x \in \bar{T}$.

Now let $s$ be a semisimple element of $G$. Then there exists a maximal $\sigma$-stable torus $\bar{S}$ of $\bar{G}$ containing $s$. Since all maximal tori of $\bar{G}$ are conjugate, we have that there exists $g \in \bar{G}$ such that $\bar{S}^{g}=\bar{T}$. Therefore $s^{g n_{0} \bar{\tau} g^{-1}}=s^{-1}$. Since $n_{0}^{\sigma}=n_{0}$ and $\bar{\tau}^{\sigma}=\bar{\tau}$ we have that $\left(g n_{0} \bar{\tau} g^{-1}\right)^{\sigma}=g^{\sigma} n_{0} \bar{\tau}\left(g^{-1}\right)^{\sigma}$. Moreover, since $\bar{S}$ is $\sigma$-stable, then for every $x \in \bar{S}$ we 
have that $x^{g n_{0} \bar{\tau} g^{-1}}=x^{g^{\sigma} n_{0} \bar{\tau}\left(g^{-1}\right)^{\sigma}}=x^{-1}$, i. e., $g n_{0} \bar{\tau} g^{-1} \bar{S}=g^{\sigma} n_{0} \bar{\tau}\left(g^{-1}\right)^{\sigma} \bar{S}$. In particular, there exists $t \in \bar{S}$ such that $g n_{0} \bar{\tau} g^{-1} t=g^{\sigma} n_{0} \bar{\tau}\left(g^{-1}\right)^{\sigma}$. In view of Lang-Steinberg Theorem [18, Theorem 10.1] there exists $y \in \bar{S}$ such that $t=y \cdot\left(y^{-1}\right)^{\sigma}$. Therefore, $g n_{0} \bar{\tau} g^{-1} y=\left(g n_{0} \bar{\tau} g^{-1} y\right)^{\sigma}$, i. e., $g n_{o} \tau g^{-1} y \in \bar{G}_{\sigma} \lambda\langle\tau\rangle$, and $s^{g n_{o} \tau g^{-1} y}=s^{-1}$. Since $O^{p^{\prime}}\left(\bar{G}_{\sigma}\right) \bar{S}_{\sigma}=\bar{G}_{\sigma}$, and $\bar{S}_{\sigma}$ is Abelian, we may find $z \in \bar{S}_{\sigma}$ such that $g n_{0} \tau g^{-1} y z \in O^{p^{\prime}}\left(\bar{G}_{\sigma}\right) \lambda\langle\tau\rangle$.

Lemma 4.6. Let $\Gamma G$ be a finite semilinear group of Lie type over a field of characteristic $p$ (we do not exclude case $\Gamma G=G$ ) and $Z(G)=\{e\}$. Assume that $B=U \lambda H$, where $H$ is a Cartan subgroup of $G$, is a $\zeta$-invariant Borel subgroup of $G$ and that $B\rangle\langle\zeta\rangle$ contains a Carter subgroup $K$ of $\Gamma G$. Consider $K_{u}=K \cap U$ and assume that $K_{u} \neq\{e\}$. Then one of the following holds:

(1) Either $\Gamma G={ }^{2} A_{2}\left(2^{2 t}\right) \lambda\langle\zeta\rangle$, or $\Gamma G={ }^{2} \widehat{A_{2}\left(2^{2 t}\right)} \lambda\langle\zeta\rangle ;|\zeta|=t$ is odd, $C_{G}(\zeta) \simeq \widehat{{ }^{2} A_{2}(2)}$, $K \cap G$ has order $2 \cdot 3$ and is isomorphic to a Carter subgroup of ${ }^{2} \widehat{A_{2}\left(2^{2}\right)}$.

(2) $G$ is defined over $G F\left(2^{t}\right), \zeta$ is a field automorphism, $|\zeta|=t$, and $K$ contains a Sylow 2-subgroup of $G_{\zeta_{2^{\prime}}}$.

(3) $G / Z(G) \simeq \mathbf{P} S L_{2}\left(3^{t}\right),|\zeta|=t$ is odd, and $K$ contains a Sylow 3 -subgroup of $G_{\zeta_{3^{\prime}}}$.

(4) $\Gamma G={ }^{2} G_{2}\left(3^{2 n+1}\right) \lambda\langle\zeta\rangle,|\zeta|=2 n+1, K \cap{ }^{2} G_{2}\left(3^{2 n+1}\right)=S \times P$, where $S$ is of order 2 and $|P|=3^{|\zeta|_{3}}$.

Proof. If $G$ is one of the following groups: $A_{1}(q), G_{2}(q), F_{4}(q),{ }^{2} B_{2}\left(2^{2 n+1}\right)$, or ${ }^{2} F_{4}\left(2^{2 n+1}\right)$, then the lemma follows from $[9$, Table] or [10, Table]. If $\Gamma G=G$ then our result follows from [6] and [10]. So we may assume that $\Gamma G \neq G$, i. e., that $\Gamma G$ contains a nontrivial field or a graph-field automorphism $\zeta$. It is convenient to say that field automorphism of even order of twisted group is a graph-field automorphism, and through the proof of the lemma we use this definition. Let $\Phi$ be the root system of $\bar{G}$. If $\Phi=C_{\ell}$, then we prove this lemma later in Theorem 5.1, so we assume that $\Phi \neq C_{\ell}$. If $\Phi=D_{4}$ and either graph-field automorphism contains a graph automorphism of order 3 , or $G \simeq{ }^{3} D_{4}\left(q^{3}\right)$ and $|\zeta| \equiv 0(\bmod 3)$, then we prove this lemma later in Theorem 6.1. Since we shall use this lemma in the proof of Theorem 7.1, after Theorems 5.1 and 6.1 , it is possible to make such additional assumptions on $G$.

Assume that $q$ is odd and $\Phi$ is one of the following types: $A_{\ell}(\ell \geqslant 2), D_{\ell}(\ell \geqslant 3), B_{\ell}(\ell \geqslant 3)$, $E_{6}, E_{7}$ or $E_{8}$. By Lemma 2.1 we have that $K U / U$ is a Carter subgroup of $B \lambda\langle\zeta\rangle \simeq H \lambda\langle\zeta\rangle$. Since $H_{\zeta} \leq Z(H \lambda\langle\zeta\rangle)$, we obtain, up to conjugation in $B$, that $H_{\zeta} \leq K$. If $\zeta$ is a field automorphism, then $\Omega(H) \leq H_{\zeta}$. In view of nilpotency of $K$ we obtain that $K_{u} \leq C_{U}(\Omega(H))$. By Lemma 3.2 it follows that $C_{U}(\Omega(H))=\{e\}$, a contradiction with $K_{u} \neq\{e\}$. If $\zeta$ is a graph-field automorphism, then $\zeta_{2} \neq e$ and $\zeta_{2^{\prime}}$ centralizes $\Omega(H)$. Thus every element of odd order of $H \lambda\langle\zeta\rangle$ centralizes $\Omega(H)$ and, up to conjugation in $B$, we have that $\Omega(H) \leq K$. Again by Lemma 3.2 we obtain a contradiction.

Assume that $G \simeq{ }^{2} G_{2}\left(3^{2 n+1}\right)$ and $\Gamma G=G \lambda\langle\zeta\rangle$. Again by Lemma 2.1 we have that $K U / U$ is a Carter subgroup of $H \lambda\langle\zeta\rangle$. Since $\left(2 n+1,3^{2 n+1}-1\right)=1$ we have that $H_{\zeta} \simeq K U / U \cap H U / U$ is of order 2. Thus $K \cap G=K_{u} \times\langle t\rangle$, where $t$ is an involution. It follows that $K_{u}=C_{G}(t) \cap G_{\zeta_{3^{\prime}}}$. Now case (4) follows from [30] and [31, Theorem 1].

Assume now that $q=2^{t}$ is even. Assume first that $\Phi$ is one of the following types: $A_{\ell}$ $(\ell \geqslant 2), D_{\ell}(\ell \geqslant 3), B_{\ell}(\ell \geqslant 3), E_{6}, E_{7}$ or $E_{8}, G$ is split, and $\zeta$ is a field automorphism. It is easy to see that for any $r \in \Phi$ there exists $s \in \Phi$ such that $\langle s, r>=1$. If $|\zeta| \neq t$, then $h_{s}(\lambda) \in H_{\zeta} \leq K$ for some $\lambda \neq 1$, hence $x_{r}(t)^{h_{s}(\lambda)}=x_{r}(t)\left(\lambda^{<s, r>} t\right)=x_{r}(\lambda t)(\mathrm{cf}$. [12, 
Proposition 6.4.1]). It follows that $K_{u} \leq C_{U}\left(H_{\zeta}\right)=\{e\}$, a contradiction. Therefore, $|\zeta|=t$, $H_{\zeta}=\{e\}$ and we obtain statement $(2)$ of the lemma.

Now assume that $\Phi$ is of type: $A_{\ell}(\ell \geqslant 2), D_{\ell}(\ell \geqslant 3)$, or $E_{6}$; and either $G$ is split and $\zeta$ is a graph-field automorphism, or $G$ is twisted. Let $\rho$ be the symmetry of the Dynkin diagram of $\Phi$ corresponding to $\tau$ and denote $r^{\rho}$ by $\bar{r}$. If $\Phi \neq A_{2}$, then for every $r \in \Phi$ there exists $s \in \Phi$ such that $s+\bar{s} \notin \Phi$ and $\langle s, r\rangle=1$. Then we proceed like in case of field automorphism, taking $h_{s}(\lambda) h_{\bar{s}}\left(\lambda^{2^{t / l}}\right)$. If $G=A_{2}\left(2^{t}\right)$ then $K \geq H_{\zeta}$ contains an element $x$ of order 3 such that $x \in{ }^{2} A_{2}\left(2^{2}\right) \leq A_{2}\left(2^{2}\right) \leq A_{2}\left(2^{t}\right)$. By using [32] or [33] one can see that $x$ is conjugate to $x^{-1}$ in $A_{2}\left(2^{2}\right)$, hence in $G$. Since the only composition factor of $C_{G}(x)$ is isomorphic to $A_{1}\left(2^{t}\right)$ (see [16, Proposition 7]), then [9, Table] and [10, Theorem 3.5] imply that $C_{G \lambda\langle\zeta\rangle}(x)$ satisfies $(*)$, a contradiction with Lemma 2.2.

Now assume that $G \simeq{ }^{2} A_{2}\left(2^{2 t}\right)$. By Lemma 2.1 we have that $K U / U$ is a Carter subgroup of $H \lambda\langle\zeta\rangle$. Now if $|\zeta|$ is even, then $H_{\zeta}$ is isomorphic to a Cartan subgroup of $A_{2}\left(2^{2 t /|\zeta|}\right)$. If $H_{\zeta}=\{e\}$, we obtain statement (2) of the lemma, if $H_{\zeta} \neq\{e\}$, then $K_{u} \leq C_{U}\left(H_{\zeta}\right)=\{e\}$, and this gives a contradiction with the condition $N_{G}\left(K_{u}\right)=B$. If $|\zeta| \neq t$ is odd, then $H_{\zeta} \leq K$ contains an element $x$ of order greater, than 3 and direct calculations show that $C_{U}(x)=\{e\}$. If $|\zeta|=t$ is odd, then we obtain statement (1) of the lemma.

\section{$5 \quad$ Carter subgroups in symplectic groups}

From now by $C$ min we denote the minimal $n$ such that $A$ is an almost simple group, $F^{*}(A)$ is a simple group of Lie type of order $n$ and $A$ contains nonconjugate Carter subgroups. We shall prove that $C \min =\infty$, i. e. that such a group $A$ does not exist. In this section we consider Carter subgroups in an almost simple group $A$ with simple socle $G=F^{*}(A) \simeq \mathbf{P} S p_{2 n}(q)$. We consider such groups here, since for groups of type $\mathbf{P} S p_{2 n}(q)$ Lemma 3.2 is not true and we use arguments slightly different from those that we use in proof of Theorem 7.1.

Theorem 5.1. Let $G$ be a finite group of Lie type with trivial centre (not necessary simple) over a field of characteristic $p$ and $\bar{G}, \sigma$ are chosen so that $\mathbf{P} S p_{2 n}\left(p^{t}\right) \simeq O^{p^{\prime}}\left(\bar{G}_{\sigma}\right) \leq G \leq \bar{G}_{\sigma}$. Choose a subgroup $A$ of $\operatorname{Aut}\left(\mathbf{P} S p_{2 n}\left(p^{t}\right)\right)$ containing $G$. Let $K$ be a Carter subgroup of $A$. Assume also that $\left|\mathbf{P} S p_{2 n}\left(p^{t}\right)\right| \leq C$ min and $A=\langle K, G\rangle$.

Then exactly one of the following statements holds:

(1) $G$ is defined over $G F\left(2^{t}\right),|\zeta|=t$, and $K=S \lambda\langle\zeta\rangle$, where $S$ is a Sylow 2-subgroup of $G_{\zeta_{2^{\prime}}}$.

(2) $G \simeq \mathbf{P} S L_{2}\left(3^{t}\right) \simeq \mathbf{P} S p_{2}\left(3^{t}\right),|\zeta|=t$ is odd, and $K=S \lambda\langle\zeta\rangle$, where $S$ is a Sylow 3-subgroup of $G_{\zeta_{3^{\prime}}}$.

(3) $p$ does not divide $|K \cap G|$ and $K$ is contained in the normalizer of a Sylow 2-subgroup of $A$.

Proof. Assume by contradiction that $K$ is a Carter subgroup of $\Gamma G$ and $K$ does not satisfy the theorem. Write $K=\left\langle x, K_{G}\right\rangle$, where $K_{G}=K \cap G \unlhd K$. If either $p \neq 3$ or $t$ is even, then our result follows from [10, Theorem 3.5]. Thus we may assume that $q=3^{t}$ and $t$ is odd. By [10, Lemma 2.2] we have that every semisimple element of odd order is conjugate to its inverse in $G$. Now, for every semisimple element $t \in G$, every non-Abelian composition factor of $C_{G}(t)$ is a simple group of Lie type (cf. [23]) of order less, than $C$ min. Therefore, for every 
non-Abelian composition factor $S$ of $C_{G}(t)$, Carter subgroups of $\operatorname{Aut}_{C_{G}(t)}(S)$ are conjugate. It follows that $C_{G}(t)$ satisfies $(*)$. Hence, by Lemma $2.2,\left|K_{G}\right|=2^{\alpha} \cdot 3^{\beta}$ for some $\alpha, \beta \geqslant 0$. If $G=\mathbf{P} \widehat{S p_{2 n}(q)}$, then by [34, Theorem 2] we have that every unipotent element is conjugate to its inverse. Since 3 is a good prime for $G$, then [35, Theorem 1.2 and 1.4] imply that, for any element $u \in G$ of order 3, all composition factors of $C_{G}(u)$ are simple groups of Lie type of order less, than $C$ min. Thus $C_{G}(u)$ satisfies $(*)$, hence, by Lemma 2.2, we obtain that $K_{G}$ is a 2-group. By Lemmas 4.3 and 4.4 every element $x \in A \backslash G$ of odd order with $\langle x\rangle \cap G=\{e\}$ centralizes some Sylow 2-subgroup of $G$. Hence $K$ contains a Sylow 2-subgroup of $A$, i. e., $K$ satisfies (3) of the theorem. Thus we may assume that $G=\mathbf{P} S p_{2 n}(q)$ and $\beta \geqslant 1$, i. e., a Sylow 3-subgroup $O_{3}\left(K_{G}\right)$ of $K_{G}$ is nontrivial. By Lemma 4.2 we obtain that $K_{G}$ is contained in some $K$-invariant parabolic subgroup $P$ of $G$ with the Levi factor $L$ and, up to conjugation in $P$, a Sylow 2-subgroup $O_{2}\left(K_{G}\right)$ of $K_{G}$ is contained in $L$. We have that $K O_{3}(P) / O_{3}(P)$ is isomorphic to $\widetilde{K}=K / O_{3}\left(K_{G}\right)$ and, by Lemma $2.1, \widetilde{K}$ is a Carter subgroup of $\langle\widetilde{K}, L\rangle$. Now $\widetilde{K} \cap L=O_{2}\left(K_{G}\right)$ is a 2-group and every element $x \in\langle\widetilde{K}, L\rangle \backslash L$ with $\langle x\rangle \cap L=\{e\}$ of odd order centralizes a Sylow 2-subgroup of $L$ (cf. Lemmas 4.3 and 4.4). Therefore $O_{2}\left(K_{G}\right)$ contains a Sylow 2-subgroup of $L$, in particular, contains a Sylow 2-subgroup of $H$. Hence, $K_{G}$ contains $\Omega(H)$. Since $K$ is nilpotent, Lemma 3.3 implies that $O_{3}\left(K_{G}\right) \leq C_{U}(\Omega(H))=\left\langle X_{r}\right| r$ is a long root $\rangle$. Since for any two long positive roots $r, s$ we have that $r+s \notin \Phi$, Chevalley commutator formulae [12, Theorem 5.2.2] implies that $\left\langle X_{r}\right| r$ is a long root $\rangle$ is Abelian.

Up to equivalence of root systems, we may suppose that $\Phi$ is contained in a Euclidean space with orthonormal basis $e_{1}, \ldots, e_{n}$, and its roots has the form $\pm e_{i} \pm e_{j}, i, j \in\{1, \ldots, n\}$ (short roots) or $\pm 2 e_{i}, i \in\{1, \ldots, n\}$ (long roots). If $\left\{r_{1}, r_{2}, \ldots, r_{n-1}, r_{n}\right\}=\left\{e_{1}-e_{2}, e_{2}-\right.$ $\left.e_{3}, \ldots, e_{n-1}-e_{n}, 2 e_{n}\right\}$ is a set of fundamental roots of $\Phi$, then long positive roots has the following form $r_{n}+2 r_{n-1}+\ldots+2 r_{k}=2 e_{k}$ for some $k$. Thus there exists a nontrivial $O_{2}\left(K_{G}\right)$ invariant subgroup $\left\langle X_{r} \mid r \in I\right\rangle=O_{p}(P) \cap\left\langle X_{r}\right| r$ is a long root $\rangle$, where $I$ is a subset of the set of long positive roots. Group $O_{2}\left(K_{G}\right)$ acts by conjugation on $\left\langle X_{r} \mid r \in I\right\rangle$, thus we obtain a representation $O_{2}\left(K_{G}\right) \rightarrow \operatorname{Sym}(I)$. Assume that there exists an orbit $\Omega$ of length greater, than 1 such that $O_{3}\left(K_{G}\right) \cap\left\langle X_{r} \mid r \in \Omega\right\rangle \neq\{e\}$. Without lost we may assume that it is $\left\{X_{2 e_{n}}, \ldots, X_{2 e_{k}}\right\}$. Since $K$ is nilpotent, then $O_{3}\left(K_{G}\right) \cap\left\langle X_{2 e_{n}}, \ldots, X_{2 e_{k}}\right\rangle$ contains an element $v=x_{2 e_{n}}(t) \cdot x_{2 e_{n-1}}(t)$. $\ldots \cdot x_{2 e_{k}}(t)$ for some $t \in G F(q)$ and it is central in $K$. Indeed, $K \cap\left\langle X_{2 e_{n}}, X_{2 e_{n-1}}, \ldots, X_{2 e_{k}}\right\rangle$ is normal in $K_{G}, \zeta$ normalizes $K \cap\left\langle X_{2 e_{n}}, X_{2 e_{n-1}}, \ldots, X_{2 e_{k}}\right\rangle$ (since $\zeta$ normalizes each of $X_{r}$ ), hence, $K \cap\left\langle X_{2 e_{n}}, X_{2 e_{n-1}}, \ldots, X_{2 e_{k}}\right\rangle$ is normal in $K$. Therefore, $Z(K) \cap\left(K \cap\left\langle X_{2 e_{n}}, X_{2 e_{n-1}}, \ldots, X_{2 e_{k}}\right\rangle\right)$ is nontrivial. Since $O_{2}\left(K_{G}\right)$ acts transitively on $\Omega$, we obtain required form of $v$. Now, either $v$, or $v^{-1}$ under $H$ is conjugate to $f=x_{2 e_{n}}(1) \cdot x_{2 e_{n-1}}(1) \cdot \ldots \cdot x_{2 e_{k}}(1)$, therefore we may assume that $v=x_{2 e_{n}}(1) \cdot x_{2 e_{n-1}}(1) \cdot \ldots \cdot x_{2 e_{k}}(1)$. We want to show that $v$ and $v^{-1}$ are conjugate in $G$. Since $n-k+1$ is even (as the order of an orbit of a 2-group), we may write $v=v_{k} \cdot v_{k+2} \cdot \ldots \cdot v_{n-1}$, where $v_{i}=x_{2 e_{i}}(1) x_{2 e_{i+1}}(1)$. Now we show that there exist $x_{k}, x_{k+2}, \ldots, x_{n-1}$ such that

$$
v_{i}^{x_{j}}=\left\{\begin{array}{ll}
v_{i}^{-1} & \text { if } i=j, \\
v_{i} & \text { if } i \neq j .
\end{array},\right.
$$

i. e., $v^{x_{k} \cdot x_{k+2} \cdot \ldots \cdot x_{n-1}}=v^{-1}$. We construct $x_{n-1}$. We may choose structure constant so that $C_{1,1, r_{n-1}, r_{n}}=1, C_{1,1, r_{n-1}, r_{n-1}+r_{n}}=1, C_{2,1, r_{n-1}, r_{n}}=-1$. Then Chevalley commutator formulae [12, Theorem 5.2.2] implies that

$$
\left(x_{r_{n}}(1) \cdot x_{r_{n}+2 r_{n-1}}(1)\right)^{x_{r_{n-1}+r_{n}}(1)}=x_{r_{n}}(1) \cdot x_{r_{n}+r_{n-1}}(-1) \cdot x_{r_{n}+2 r_{n-1}}(-1) .
$$


Consider a reflection $w$ in the root $e_{n-1}-e_{n}$. We may choose its preimage $x$ in $G$ so that

$$
\left(x_{r_{n}}(1) \cdot x_{r_{n}+r_{n-1}}(-1) \cdot x_{r_{n}+2 r_{n-1}}(-1)\right)^{x}=x_{r_{n}}(-1) \cdot x_{r_{n}+r_{n-1}}(-1) \cdot x_{r_{n}+2 r_{n-1}}(1)
$$

At the end, again by using Chevalley commutator formulae, we have

$$
\left(x_{r_{n}}(-1) \cdot x_{r_{n}+r_{n-1}}(-1) \cdot x_{r_{n}+2 r_{n-1}}(1)\right)^{x_{r_{n-1}+r_{n}}(1)}=x_{r_{n}}(-1) \cdot x_{r_{n}+2 r_{n-1}}(-1),
$$

i. e., $x_{n-1}=x_{r_{n-1}+r_{n}}(1) \cdot x \cdot x_{r_{n-1}+r_{n}}(1)$. Clearly $x_{n-1}$ commutes with all $v_{i}$ for $k \leqslant i \leqslant n-3$. We construct $x_{n-3}, \ldots, x_{k}$ in the same way. Thus we obtain that $v$ and $v^{-1}$ are conjugate in $G$. Again by [35, Theorem 1.2 and 1.4] we have that all non-Abelian composition factors of $C_{G}(v)$ are simple groups of Lie type of order less, than $C$ min. Therefore, $C_{G}(v)$ satisfies $(*)$, a contradiction with Lemma 2.2. Hence every $O_{2}\left(K_{G}\right)$-orbit $\Omega$ of $\operatorname{Sym}(I)$ with $O_{3}\left(K_{G}\right) \cap\left\langle X_{r}\right| r \in$ $\Omega\rangle \neq\{e\}$ has length 1. Therefore, for some $i, O_{3}\left(K_{G}\right) \cap X_{2 e_{i}} \neq\{e\}$ is normal in $K$. Without lost we may assume that $i=1$ and $X_{2 e_{1}}=X_{r_{0}}$, where $r_{0}$ is the highest root of $\Phi$. Up to conjugation in $G$ we may assume that $x_{r_{0}}(1) \leq Z(K)$. Thus $K_{G} \leq C_{G}\left(x_{r_{0}}(1)\right)=P$, where $P$ is the parabolic subgroup obtaining by removing $r_{1}$ from the set of fundamental roots. The Levi factor $L$ of $P$ is known to have the following structure $C_{n-1}(q) * S$, where $S=Z(L)$. Now, up to conjugation in $P, O_{2}\left(K_{G}\right)$ is the Sylow 2-subgroup of $C_{n-1}(q)$ and $K S O_{3}(P) / S O_{3}(P)$ is a Carter subgroup of $C_{n-1}(q) \lambda\langle\zeta\rangle$. Since $t$ is odd we have that $O_{2}\left(K_{G}\right)$ is a Sylow 2-subgroup of $C_{n-1}(q)$ and Lemma 4.3 implies that $O_{2}\left(K_{G}\right)$ is a Sylow 2-subgroup of $\left(C_{n-1}(q)\right)_{\zeta}=C_{n-1}(3)$. But, by Lemma 3.4, $N_{C_{n-1}(3)}\left(O_{2}\left(K_{G}\right)\right) \neq O_{2}\left(K_{G}\right) C_{C_{n-1}(3)}\left(O_{2}\left(K_{G}\right)\right)$, hence, by Lemma $2.3, K$ is not a Carter subgroup of $C_{n-1}(q) \lambda\langle\zeta\rangle$. This final contradiction completes the proof.

\section{Groups with triality automorphism}

Theorem 6.1. Let $G$ be either $D_{4}(q)$, or ${ }^{3} D_{4}\left(q^{3}\right)$. Assume that $\tau$ is a graph automorphism of $G$ of order 3 (in case of ${ }^{3} D_{4}\left(q^{3}\right)$ this is an automorphism, which has the set of stable points isomorphic to $\left.D_{4}(q)\right)$. Denote by $A_{1}$ the subgroup of $\operatorname{Aut}(G)$ generated by all automorphisms, except $\tau$. Let $A \leq \operatorname{Aut}(G)$ be such that $A \not \leq A_{1}$, and let $K$ be a Carter subgroup of $A$. Assume also that $|G| \leqslant C$ min.

Then $\tau \in K, K \cap A_{1}$ is a Sylow 2-subgroup of $C_{A}(\tau) \simeq \Gamma G_{2}(q)$ and $\Gamma G_{2}(q) / G_{2}(q)$ is a 2-group. A Sylow 2-subgroup $S$ of $\Gamma G_{2}(q)$ satisfies $N_{\Gamma G_{2}(q)}(S)=S C_{\Gamma G_{2}(q)}(S)$ (i. e. satisfies Lemma 2.3) if either $q$ is odd, or $q$ is even, $q=2^{2^{t}}$, and $\left|\Gamma G_{2}(q): G_{2}(q)\right|=2^{t}$.

Proof. In view of [36, Theorem $1.2($ vi)] we have that every element of $G$ is conjugate to its inverse. By [23] and [35, Theorems 1.2 and 1.4] we obtain that for any element $t \in G$ of odd prime order, all non-Abelian composition factors of $C_{G}(t)$ are simple groups of Lie type of order less, then $C$ min. Thus, $C_{A}(t)$ satisfies $(*)$ and Lemma 2.2 implies that $K_{G}=K \cap G$ is a 2-group. Now Lemma 4.4 implies that all field or graph-field automorphisms of odd order of $G$ are conjugate under $G$. Since the centralizer of every field or graph-field automorphism in $G$ is a group of Lie type of order less, than $C$ min, we again may apply Lemma 2.2 and obtain that $A$ does not contain field automorphism of odd order. Therefore, $K$ contains an element $s$ of order 3 such that $\langle s\rangle \cap A_{1}=\{e\}, G \lambda\langle s\rangle=G \lambda\langle\tau\rangle$, and $K \cap A_{1}$ is a 2-group.

There exists two non-conjugate cyclic subgroups of order 3: $\langle\tau\rangle$ and $\langle x\rangle$ such that $\langle\tau\rangle \cap A_{1}=$ $\langle x\rangle \cap A_{1}=\{e\}$ and $G \lambda\langle s\rangle=G \lambda\langle\tau\rangle$ (see [29, (9-1)]). Hence, either $\tau \in K$, or $x \in K$. Assume that $q \neq 3^{t}$. In the first case we obtain the statement of the theorem, in the second case we have that $K \leq\left\langle K, C_{G}(x)\right\rangle$. But $C_{G}(x) \simeq \mathbf{P} G L_{3}^{\varepsilon}(q)$, where $q \equiv \varepsilon= \pm 1(\bmod 3)$ and 
$\mathbf{P} G L_{3}^{+1}(q)=\mathbf{P} G L_{3}(q), \mathbf{P} G L_{3}^{-1}(q)=\mathbf{P} G U_{3}(q)$. In view of [3], we have that $K$ contains a Sylow 2-subgroup of $\mathbf{P} G L_{3}^{\varepsilon}(q)$. Since the normalizer of a Sylow 2-subgroup of $\mathbf{P} G L_{3}^{\varepsilon}(q)$ is nilpotent, we have that $K$ contains the normalizer of a Sylow 2-subgroup of $\mathbf{P} G L_{3}^{\varepsilon}(q)$. But, under our conditions, this normalizer contains a cyclic subgroup $q-\varepsilon$ and 3 divides $q-\varepsilon$. Therefore, $K \cap G$ contains an element of odd order, a contradiction. Thus this second case is impossible.

Assume now that $q=3^{t}$. Then $C_{G}(\tau) \simeq G_{2}(q)$ and we obtain the theorem. In the second case $C_{G}(x) \simeq S L_{2}(q) \curlywedge U$, where $U$ is a 3-group and $Z\left(C_{G}(x)\right) \cap U \neq\{e\}$, a contradiction with Lemma 2.2.

\section{Carter subgroups in semilinear groups of Lie type}

Theorem 7.1. Let $G$ be a finite group of Lie type ( $G$ is not necessary simple) over a field of characteristic $p$ and $\bar{G}, \sigma$ are chosen so that $O^{p^{\prime}}\left(\bar{G}_{\sigma}\right) \leq G \leq \bar{G}_{\sigma}$. Assume also that $G \nsucceq{ }^{3} D_{4}\left(q^{3}\right)$. Choose a subgroup $A$ of $\operatorname{Aut}\left(O^{p^{\prime}}\left(\bar{G}_{\sigma}\right)\right)$ containing $G$ and assume that $A$ is not contained in subgroup $A_{1}$ defined in Theorem 6.1, if $G=D_{4}(q)$. Let $K$ be a Carter subgroup of $A$ and assume that $A=K G$. Assume also that $|G| \leq C$ min.

Then exactly one of the following statements holds:

(1) $\Gamma G={ }^{2} A_{2}\left(2^{2 t}\right) \lambda\langle\zeta\rangle,|\zeta|=t$ is odd, $K \cap{ }^{2} A_{2}\left(2^{2 t}\right)$ has order $2 \cdot 3$ and is isomorphic to a Carter subgroup of ${ }^{2} A_{2}\left(2^{2}\right)$.

(2) $G$ is defined over $G F\left(2^{t}\right), \zeta$ is a field automorphism, $|\zeta|=t$, and $K=S \lambda\langle\zeta\rangle$, where $S$ is a Sylow 2-subgroup of $G_{\zeta_{2^{\prime}}}$.

(3) $G \simeq \mathbf{P} S L_{2}\left(3^{t}\right),|\zeta|=t$ is odd, and $K=S \lambda\langle\zeta\rangle$, where $S$ is a Sylow 3-subgroup of $G_{\zeta_{3^{\prime}}}$.

(4) $\Gamma G={ }^{2} G_{2}\left(3^{2 n+1}\right) \lambda\langle\zeta\rangle,|\zeta|=2 n+1$, and $K \cap{ }^{2} G_{2}\left(3^{2 n+1}\right)=S \times P$, where $S$ is of order 2 and $|P|=3^{|\zeta|_{3}}$.

(5) $p$ does not divide $|K \cap G|$ and $K$ contains a Sylow 2-subgroup of $A$. Note that in view of Lemmas 2.6, 4.3 and 4.4 a Sylow subgroup $S$ of $A$ satisfies $N_{A}(S)=S C_{A}(S)$ if $N_{G}(S \cap$ $G)=(S \cap G) C_{G}(S \cap G)$.

In particular, Carter subgroups of $A$ are conjugate.

Note that after prooving this theorem we can do not demand that $A=K G$ and $|A| \leqslant C$ min.

We shall prove this theorem in the following way. If $G=C_{n}(q)$, then the theorem follows from Theorem 5.1. If $G=A$ then the theorem follows from [6] and [10]. Thus we may assume that $G \neq C_{n}(q)$ and $G \neq A$. Then first of all, it is possible to check by direct computation, that $G$ and $K$ can satisfy precisely one of the statements of the theorem, i. e., if (3) holds, and $S$ is a Sylow 2-subgroup of $A$, then, by Lemma 3.4, $N_{A}(S) \neq S C_{A}(S)$, hence (5) is not true. If (4) holds then by [30], a Sylow 2-subgroup $S$ of $A$ does not satisfy $N_{A}(S)=S C_{A}(S)$, so by Lemma 2.3, statement (5) of the theorem is not true.

Assume that the theorem is false and $A$ is a counterexample with $|G|$ minimal. Clearly this implies that $Z(G)$ is trivial. Let $K$ be a Carter subgroup of $A$. First we show that if $p$ divides $|K|$, then one of (1)-(4) of the theorem holds. Then we show that if $p$ does not divide $|K|$, then $K$ contains a Sylow 2-subgroup of $A$. Since both of these steps are quite complicated, we divide them into two sections. Note also that by [23], for every semisimple element $t \in G$, all 
non-Abelian composition factors of $C_{G}(t)$, hence of $C_{A}(t)$ are simple groups of Lie type of order less, than $C$ min. Hence $C_{A}(t)$ satisfies $(*)$. In order to apply Lemma 2.2 we shall use this fact without future references. Further, for every non-Abelian composition factor $S \simeq D_{4}(q)$ of a reductive subgroup of maximal rank $R$ of $G$, we have that $\operatorname{Aut}_{A}(S)$ is contained in subgroup $A_{1} \leq \operatorname{Aut}(S)$, i. e. satisfies conditions of the theorem.

\section{Carter subgroups of order divisible by the character- istic}

Denote $K \cap G$ by $K_{G}$. If $A$ contains a graph automorphism $\tau$ of $O^{p^{\prime}}\left(\bar{G}_{\sigma}\right)$, then every semisimple element of odd order is conjugate to its inverse in $A$ (cf. Lemma 4.5). By Lemma 2.2 we obtain that $\left|K_{G}\right|$ is divisible only by 2 and $p$. If $p=2$, then we obtain that $K_{G}$ is a 2group, it is contained in a proper $K$-invariant parabolic subgroup $P$ of $G$ and by Lemma $2.1 \mathrm{KO}_{2}(\mathrm{P}) / \mathrm{O}_{2}(\mathrm{P})$ is a Carter subgroup of $K P / \mathrm{O}_{2}(P)$. Since $K_{G} \leq \mathrm{O}_{2}(P)$, it follows that $\left(K \mathrm{O}_{2}(P) / \mathrm{O}_{2}(P)\right) \bigcap\left(P / O_{2}(P)\right)=\{1\}$. Hence $P$ is a Borel subgroup of $G$, otherwise we would have $C_{P / O_{2}(P)}\left(K O_{2}(P) / O_{2}(P)\right) \neq\{1\}$, a contradiction with the fact that $K O_{2}(P) / O_{2}(P)$ is a Carter subgroup of $K P / O_{2}(P)$. Thus $P$ is a Borel subgroup and the theorem follows from Lemma 4.6. Now if $p \neq 2$, then again $K_{G}$ is contained in a proper parabolic subgroup $P$ of $G$ such that $O_{p}\left(K_{G}\right) \leq O_{p}(P)$ and $O_{2}\left(K_{G}\right) \leq L$. Then Lemmas 4.3 and 4.4 implies that $\Omega(H) \leq K$. Now Lemma 3.2 implies that $O_{p}\left(K_{G}\right) \leq C_{U}(\Omega(H))=\{e\}$. Therefore $K \cap G$ is a 2-group. By Lemmas 4.3 and 4.4 every element $x \in A \backslash G$ of odd order such that $\langle x\rangle \cap G=\{e\}$ centralizes some Sylow 2-subgroup of $G$. Hence $K$ contains a Sylow 2 -subgroup of $A$, i. e., $K$ satisfies (5) of the theorem. Hence we may assume that $A=\Gamma G$.

Recall that we are in the conditions of Theorem 7.1, $\Gamma G$ is supposed to be a counterexample to the theorem with $|G|$ minimal and $K$ is a Carter subgroup of $\Gamma G$ such that $p$ divides $\left|K_{G}\right|$. We have that $K=\left\langle\zeta^{k} g, K_{G}\right\rangle$. Since $|G| \leqslant C m i n$, Lemma 2.1 implies that $K G / G$ is a Carter subgroup of $\Gamma G / G$. Therefore $\left|\zeta^{k}\right|=|\zeta|$ and we may assume that $k=1$.

In view of Lemma 4.2 there exists a proper $\sigma$ - and $\bar{x}$-invariant parabolic subgroup $\bar{P}$ of $\bar{G}$ such that $O_{p}\left(K_{G}\right) \leq R_{u}(\bar{P})$ and $K_{G} \leq \bar{P}$. In particular, $\bar{P}$ and $\bar{P}^{\bar{\zeta}}$ are conjugate in $\bar{G}$. Let $\Phi$ be the root system of $\bar{G}$ and $\Pi$ be a set of fundamental roots of $\Phi$. In view of $[12$, Proposition 8.3.1] $\bar{P}$ is conjugate to some $\bar{P}_{J}=\bar{B} \cdot \bar{N}_{J} \cdot \bar{B}$, where $J$ is a subset of $\Pi$ and $\bar{N}_{J}$ is a complete preimage of $W_{J}$ in $\bar{N}$ under the natural homomorphism $\bar{N} / \bar{T} \rightarrow W$. Now $\bar{P}_{J}$ is $\varphi$-invariant, hence $\bar{P}_{J}^{\bar{\zeta}}=\bar{P}_{J}^{\bar{\gamma}^{\varepsilon}}$. Consider the symmetry $\rho$ of the Dynkin diagram of $\Phi$ corresponding to $\bar{\gamma}$. Let $\bar{J}$ be the image of $J$ under $\rho$. Clearly $\bar{P}_{J}^{\bar{\gamma}}=\bar{P}_{\bar{J}}$. Since $\bar{P}$ and $\bar{P}^{\bar{\zeta}}$ are conjugate in $\bar{G}$ we obtain that $\bar{P}_{J}$ and $\bar{P}_{J}^{\bar{\zeta}}$ are conjugate in $\bar{G}$. In view of [12, Theorem 8.3.3] it follows that either $\varepsilon=0$, or $J=\bar{J}$; i. e., $\bar{P}_{J}$ is $\bar{\zeta}$-invariant.

Now we have that $\bar{P}^{\bar{y}}=\bar{P}_{J}$. So $\langle\bar{\zeta} g, \bar{P}\rangle^{\bar{y}}=\left\langle(\bar{\zeta} g)^{\bar{y}}, \bar{P}_{J}\right\rangle$ and $\bar{P}_{J}^{(\bar{\zeta} g)^{\bar{y}}}=\bar{P}_{J}$. It follows

$$
(\bar{\zeta} g)^{\bar{y}}=\bar{y}^{-1} \bar{\zeta} g \bar{y}=\bar{\zeta}\left(\bar{\zeta}^{-1} \bar{y}^{-1} \bar{\zeta} g \bar{y}\right)=\bar{\zeta} \cdot h
$$

where $h=\left(\bar{\zeta}^{-1} \bar{y}^{-1} \bar{\zeta} g \bar{y}\right) \in \bar{G}$. Since $\bar{P}_{J}^{\bar{\zeta}}=\bar{P}_{J}=\bar{P}_{J}^{h^{-1}}$ we obtain that $h \in N_{\bar{G}}\left(\bar{P}_{J}\right)$. By [12, Theorem 8.3.3], $N_{\bar{G}}\left(\bar{P}_{J}\right)=\bar{P}_{J}$, thus $\langle\bar{\zeta} g, \bar{P}\rangle^{y}=\left\langle\bar{\zeta}, \bar{P}_{J}\right\rangle$. Now both $\bar{P}$ and $\bar{P}_{J}$ are $\sigma$-invariant. Hence $\bar{y} \sigma\left(\bar{y}^{-1}\right) \in N_{\bar{G}}(\bar{P})=\bar{P}$. Therefore, by Lang-Steinberg Theorem [18, Theorem 10.1] we may assume that $\bar{y}=\sigma(\bar{y})$, i. e. $\bar{y} \in \bar{G}_{\sigma}$. Since $\bar{G}_{\sigma}=\bar{T}_{\sigma} \cdot O^{p^{\prime}}\left(\bar{G}_{\sigma}\right)$ and $\bar{T} \leq \bar{P}_{J}$, then we may assume that $\bar{y} \in O^{p^{\prime}}\left(\bar{G}_{\sigma}\right)$. Thus, up to conjugation in $G$, we may assume that $\bar{K} \leq\left\langle\bar{\zeta}, \bar{P}_{J}\right\rangle=$ 
$\bar{P}_{J} \lambda\langle\bar{\zeta}\rangle$ and $K \leq\left(\bar{P}_{J} \cap G\right) \lambda\langle\zeta\rangle=P_{J} \lambda\langle\zeta\rangle$. Further if $\bar{L}_{J}=\left\langle\bar{T}, \bar{X}_{r} \mid r \in J \cup-J\right\rangle$, then $\bar{L}_{J}$ is a $\sigma$ and $\bar{\zeta}$-invariant Levi factor of $\bar{P}_{J}$ and $L_{J}=\bar{L}_{J} \cap G$ is a $\zeta$-invariant Levi factor of $P_{J}$. Lemma 2.1 implies that $K O_{p}\left(P_{J}\right) / O_{p}\left(P_{J}\right)=X$ is a Carter subgroup of $L_{J} \lambda\langle\zeta\rangle$ and $K_{1} Z\left(L_{J}\right) / Z\left(L_{J}\right)=\widetilde{X}$ is a Carter subgroup of $\left(L_{J} \lambda\langle\zeta\rangle\right) / Z\left(L_{J}\right)$. Recall that $K=\left\langle\zeta g, K_{G}\right\rangle$, hence, if $v$ and $\tilde{v}$ are the images of $g$ under the natural homomorphisms $\omega: P_{J} \lambda\langle\zeta\rangle \rightarrow L_{J} \lambda\langle\zeta\rangle \simeq\left(P_{J} \lambda\langle\zeta\rangle\right) / O_{p}\left(P_{J}\right)$ and $\tilde{\omega}: P_{J} \lambda\langle\zeta\rangle \rightarrow\left(P_{J} \lambda\langle\zeta\rangle\right) / Z\left(L_{J}\right) O_{p}\left(P_{J}\right) \simeq\left(L_{J} \lambda\langle\zeta\rangle\right) / Z\left(L_{J}\right)$, then $X=\left\langle\zeta v, K_{G}^{\omega}\right\rangle$ and $\widetilde{X}=$ $\left\langle\zeta \tilde{v}, K_{G}^{\tilde{\omega}}\right\rangle$. Note that $O_{p}(P)$ and $Z\left(L_{J}\right)$ are characteristic subgroups of $P$ and $L_{J}$ respectively, hence we may consider $\zeta$ as an automorphism of $L_{J} \simeq P / O_{p}(P)$ and $\widetilde{L}=L_{J} / Z\left(L_{J}\right)$. Note also that all non-Abelian composition factors of $P$ are simple groups of Lie type of order less than $C m i n$, hence $P \lambda\langle\zeta\rangle$ satisfies (*). Thus we may apply Lemma 2.1 to $\widetilde{L} \lambda\langle\zeta\rangle$.

If $P_{J}$ is a Borel subgroup of $G$, we use Lemma 4.6. So we may assume that $L_{J} \neq Z\left(L_{J}\right)$, i. e., that $P_{J}$ is not a Borel subgroup of $G$. Then $L_{J}=H\left(G_{1} * \ldots * G_{k}\right)$, where $G_{i}$ are subsystem subgroups of $G$ and $H$ is a Cartan subgroup of $G$. Let $\zeta=\zeta_{2} \cdot \zeta_{2^{\prime}}$ be the product of 2- and $2^{\prime}$-parts of $\zeta$. Now $\zeta_{2^{\prime}}=\varphi^{k}$, for some $k$, is a field automorphism (recall that we do not consider the triality automorphism) and it normalizes every $G_{i}$, since $\varphi$ normalizes every $G_{i}$. Moreover, in view of Lemma 4.3, we have that $\zeta_{2^{\prime}}$ centralizes a Sylow 2-subgroup of $H$. In particular, it centralizes a Sylow 2-subgroup of $Z\left(L_{J}\right) \leq H$. Therefore, any element of odd order of $\langle\zeta\rangle\left\langle L_{J}\right.$ centralizes a Sylow 2-subgroup of $Z\left(L_{J}\right)$.

Now $\widetilde{L}=\left(\mathbf{P} G_{1} \times \ldots \times \mathbf{P} G_{k}\right) \widetilde{H}$, where $\widetilde{H}=H^{\omega_{2}}$ and $\mathbf{P} G_{1}, \ldots, \mathbf{P} G_{k}$ are canonical finite groups of Lie type with trivial centre. Denote by $M_{i}=C_{\widetilde{L}}\left(G_{i}\right)$, clearly $M_{i}=\left(\mathbf{P} G_{1} \times \ldots \times\right.$ $\left.\mathbf{P} G_{i-1} \times \mathbf{P} G_{i+1} \times \ldots \times \mathbf{P} G_{k}\right) C_{\widetilde{H}}\left(G_{i}\right)$; denote by $L_{i}$ the factor group $\widetilde{L} / M_{i}$ and by $\pi_{i}$ corresponding natural homomorphism. Then $L_{i}$ is a finite group of Lie type and $\mathbf{P} G_{i} \leq L_{i} \leq \widehat{\mathbf{P}}_{i}$. Denote by $M_{i, j}=C_{\widetilde{L}}\left(\mathbf{P} G_{i} \times \mathbf{P} G_{j}\right)$, then $M_{i, j}=\left(\mathbf{P} G_{1} \times \ldots \times \mathbf{P} G_{i-1} \times \mathbf{P} G_{i+1} \times \ldots \times \mathbf{P} G_{j-1} \times\right.$ $\left.\mathbf{P} G_{j+1} \times \ldots \times \mathbf{P} G_{k}\right) C_{\widetilde{H}}\left(\mathbf{P} G_{i} \times \mathbf{P} G_{j}\right)$; denote by $\pi_{i, j}$ corresponding natural homomorphism $\widetilde{L} \rightarrow \widetilde{L} / M_{i, j}$. If $M_{i}$ (respectively $M_{i, j}$ ) is $\zeta$-invariant, then $M_{i}$ (resp. $M_{i, j}$ ) is normal in $\widetilde{L} \lambda\langle\zeta\rangle$ and we denote by $\delta_{i}$ (resp. $\delta_{i, j}$ ) the natural homomorphism $\delta_{i}: \widetilde{L} \lambda\langle\zeta\rangle \rightarrow(\widetilde{L} \lambda\langle\zeta\rangle) / M_{i}$ $\left(\delta_{i, j}: \widetilde{L} \lambda\langle\zeta\rangle \rightarrow(\widetilde{L} \lambda\langle\zeta\rangle) / M_{i, j}\right)$.

Now consider $\zeta_{2}$. Since $\zeta_{2}^{2}$ is a field automorphism, there can be two cases: either $\zeta_{2}$ normalizes $\mathbf{P} G_{i}$, or $\zeta_{2}^{2}$ normalizes $\mathbf{P} G_{i}$ and $\mathbf{P} G_{i}^{\zeta_{2}}=\mathbf{P} G_{j}$ for some $j \neq i$. Consider these two cases separately.

Case 1, $\zeta_{2}$ normalizes $\mathbf{P} G_{i}$. Then $\zeta_{2}$ normalizes $M_{i}$, and Lemma 2.1 implies that $\tilde{X}^{\delta_{i}}=K_{i}$ is a Carter subgroup of $L_{i} \lambda\langle\zeta\rangle$. Since $L_{i} \lambda\langle\zeta\rangle$ is a semilinear group of Lie type satisfying the conditions of Theorem 7.1, $\left|L_{i}\right|<|G|$, and $p$ does not divide $\left|K_{i}\right|$, we have that $K_{i}$ contains a Sylow 2-subgroup $S_{i}$ of $L_{i} \lambda\langle\zeta\rangle$ (in particular, $p=2$ ) and, by Lemma 2.3, $N_{L_{i} \lambda\langle\zeta\rangle}\left(S_{i}\right)=$ $S_{i} C_{L_{i} \lambda\langle\zeta\rangle}\left(S_{i}\right)=S_{i} \times O\left(N_{L_{i} \lambda\langle\zeta\rangle}\left(S_{i}\right)\right)$.

Case 2, $\zeta_{2}^{2}$ normalizes $\mathbf{P} G_{i}$ and $\mathbf{P} G_{i}^{\zeta_{2}}=\mathbf{P} G_{j}$. Then $M_{i, j}$ is normal in $\widetilde{L} \lambda\langle\zeta\rangle$. We want to show that if $S_{i, j}$ is a Sylow 2-subgroup of $(\widetilde{L})^{\pi_{i, j}} \lambda\langle\zeta\rangle$, then $N_{(\widetilde{L})^{\pi_{i, j}} \lambda\langle\zeta\rangle}\left(S_{i, j}\right)=$ $S_{i, j} C_{(\widetilde{L})^{\pi_{i, j}} \lambda\langle\zeta\rangle}\left(S_{i, j}\right)=S_{i, j} \times O\left(N_{(\widetilde{L})^{\pi_{i, j}} \lambda\langle\zeta\rangle}\left(S_{i}\right)\right)$. Since $M_{i, j}$ is a normal subgroup of $\widetilde{L} \lambda\langle\zeta\rangle$, then, by Lemma $2.1,(\widetilde{X})^{\delta_{i, j}}$ is a Carter subgroup of $(\widetilde{L} \lambda\langle\zeta\rangle)^{\delta_{i, j}}$. Thus we may assume that $\widetilde{L}=\mathbf{P} G_{1} \times \mathbf{P} G_{2}$ and $\left(\mathbf{P} G_{1}\right)^{\zeta}=\mathbf{P} G_{2}$. Now we are in the condition of Lemma 2.5, namely, we have a finite group $\widetilde{G}=\widetilde{X}\left(\mathbf{P} G_{1} \times \mathbf{P} G_{2}\right)$, where $\mathbf{P} G_{1} \simeq \mathbf{P} G_{2}$ has trivial centre. Then $\operatorname{Aut}_{\tilde{X}}\left(\mathbf{P} G_{1}\right)$ is a Carter subgroup of $\operatorname{Aut}_{\widetilde{G}}\left(\mathbf{P} G_{1}\right)$. Now $\mathbf{P} G_{1}$ is a canonical finite group of Lie type and $\mathbf{P} G_{1} \leq \operatorname{Aut}_{\widetilde{G}}\left(\mathbf{P} G_{1}\right) \leq \operatorname{Aut}\left(\mathbf{P} G_{1}\right)$, i. e., $\operatorname{Aut}_{\widetilde{G}}\left(\mathbf{P} G_{1}\right)$ satisfies conditions of Theorem 7.1 and $\tilde{X} \cap\left(\mathbf{P} G_{1} \times \mathbf{P} G_{2}\right)$ is not divisible by the characteristic. By induction, $\operatorname{Aut}_{\tilde{X}}\left(\mathbf{P} G_{1}\right)$ contains 
a Sylow 2-subgroup of $\operatorname{Aut}_{\widetilde{G}}\left(\mathbf{P} G_{1}\right)$ (in particular, $p=2$ ). The same arguments show that $\operatorname{Aut}_{\widetilde{X}}\left(\mathbf{P} G_{2}\right)$ contains a Sylow 2-subgroup of Aut $\widetilde{G}\left(\mathbf{P} G_{2}\right)$. Now consider $N_{\widetilde{G}}\left(\mathbf{P} G_{1}\right)=N_{\widetilde{G}}\left(\mathbf{P} G_{2}\right)$. Let $S$ be a Sylow 2-subgroup of $N_{\widetilde{G}}\left(\mathbf{P} G_{1}\right)$. Since $C_{\widetilde{G}}\left(\mathbf{P} G_{1}\right) \cap C_{\widetilde{G}}\left(\mathbf{P} G_{2}\right)=\{1\}$, Lemma 2.4 implies that $N_{N_{\widetilde{G}}\left(\mathbf{P} G_{1}\right)}(S)=S C_{N_{\widetilde{G}}\left(\mathbf{P} G_{1}\right)}(S)$. Now $\left|\widetilde{G}: N_{\widetilde{G}}\left(\mathbf{P} G_{1}\right)\right|=2$, thus, by Lemma 2.6, we obtain that $N_{\widetilde{G}}(\widetilde{S})=\widetilde{S} C_{N_{\widetilde{G}}\left(\mathbf{P} G_{1}\right)}(\widetilde{S})$ for a Sylow 2-subgroup $\widetilde{S}$ of $\widetilde{G}$. Hence, if $S_{i, j}$ is a Sylow 2-subgroup of $\left.\left(\mathbf{P} G_{i} \times \mathbf{P} G_{j}\right)\right\rangle\langle\zeta\rangle$, then

$$
N_{\left(\mathbf{P} G_{i} \times \mathbf{P} G_{j}\right) \lambda\langle\zeta\rangle}\left(S_{i, j}\right)=S_{i, j} C_{\left(\mathbf{P} G_{i} \times \mathbf{P} G_{j}\right) \lambda\langle\zeta\rangle}\left(S_{i, j}\right)=S_{i, j} \times O\left(N_{\left(\mathbf{P} G_{i} \times \mathbf{P} G_{j}\right) \lambda\langle\zeta\rangle}\left(S_{i, j}\right)\right) .
$$

Now we show that for a Sylow 2-subgroup $S$ of $L_{J} \lambda\langle\zeta\rangle$ we have that $N_{L_{J} \lambda\langle\zeta\rangle}(S)=$ $S C_{L_{J} \lambda\langle\zeta\rangle}(S)$. Since $\widetilde{L} \neq\{e\}$, then, as we noted above, $p \neq 2$. Consider an element $x \in$ $N_{L_{J} \lambda\langle\zeta\rangle}(S)$ of odd order. We need to prove that $x$ centralizes $S$. As we noted above, any element of odd order of $L_{J} \lambda\langle\zeta\rangle$ centralizes $S \cap Z\left(L_{J}\right)$, hence if $x$ centralizes $S /\left(S \cap Z\left(L_{J}\right)\right)$, then $x$ centralizes $S$. Now either $M_{i}$ is normal in $\widetilde{L} \lambda\langle\zeta\rangle$, or $M_{i, j}$ is normal in $\widetilde{L} \lambda\langle\zeta\rangle$ and $\left(\cap_{i} M_{i}\right) \cap\left(\cap_{i, j} M_{i, j}\right)=\{1\}$. Moreover, as we proved above $x^{\delta_{i}}$ centralizes $S M_{i} / M_{i}$, and $x^{\delta_{i, j}}$ centralizes $S M_{i, j} / M_{i, j}$. By Lemma 2.4 we obtain that $x$ centralizes $S$.

Thus $N_{L_{J} \lambda\langle\zeta\rangle}(S)=S C_{L_{J} \lambda\langle\zeta\rangle}(S)$, by Lemma 2.3 there exists a Carter subgroup $F$ of $L_{J} \lambda\langle\zeta\rangle$ containing $S$. Since $L_{J} \lambda\langle\zeta\rangle$ satisfies $(*)$, Theorem 1.1 implies that $K$ and $F$ are conjugate, i. e. $K$ contains a Sylow 2-subgroup of $L_{J} \lambda\langle\zeta\rangle$. In particular, $\Omega(H) \leq K$ and $\Omega(H)$ centralizes $K_{p}=K \cap O_{p}\left(P_{J}\right) \neq\{e\}$. A contradiction with Lemma 3.2.

\section{Carter subgroups of order not divisible by the charac- teristic}

Again we are in the conditions of Theorem 7.1. If $A$ contains a graph automorphism $\gamma$, or $\Phi(\bar{G}) \neq A_{n}, D_{2 n+1}, E_{6}$, then by Lemma 4.5 and [10, Lemma 2.2] we obtain that every semisimple element of $G$ is conjugate to its inverse. By Lemma 2.2 we have that $K_{G}=K \cap G$ is a 2-group. By Lemmas 4.3 and 4.4 we obtain that a $2^{\prime}$-part of $K$ centralizes a Sylow 2-subgroup of $G$, hence $K_{G}$ is a Sylow 2-subgroup of $G$ and $K$ contains a Sylow 2-subgroup of $A$. Thus Theorem 7.1 is true in this case. So we may assume that $A=\Gamma G=\langle\zeta, G\rangle$ is a semilinear group of Lie type, $K=\left\langle\zeta^{k} g, K_{G}\right\rangle$ is a Carter subgroup of $\Gamma G$, and $\Phi(\bar{G}) \in\left\{A_{n}, D_{2 n+1}, E_{6}\right\}$. Like in the previous section we may assume that $k=1$. Since $G_{\zeta}$ is nontrivial we have that $K_{G}$ is nontrivial also. Therefore $Z(K) \cap K_{G}$ is nontrivial. Consider an element $x \in Z(K) \cap K_{G}$ of prime order. Then $K \in C_{\Gamma G}(x)=\left\langle\zeta g, C_{G}(x)\right\rangle$. Now $C_{\bar{G}}(x)^{0}=\bar{C}$ is a connected $\sigma$-stable reductive subgroup of maximal rank of $\bar{G}$. Moreover $\bar{C}$ is a characteristic subgroup of $C_{\bar{G}}(x)$ and $C_{\bar{G}}(x) / \bar{C}$ is isomorphic to a subgroup of $\Delta$ (see [25, F, $\S 4$ and Proposition 5]). Thus $K$ is contained in $\langle K, C\rangle$, where $C=\bar{C} \cap G$. Moreover $C=\bar{C} \cap G=T\left(G_{1} * \ldots * G_{m} * S\right)$ is normal in $C_{\Gamma G}(x)$ and $K_{G} C / C$ is isomorphic to a subgroup of $\Delta$. There can be two cases:

$m=0$ and $|x|>2$, i. e., $C=T=S$ is a maximal torus. Then $\bar{T}$ is $\bar{\zeta} g$-stable. In view of Lemma 3.6 we obtain that there exists $h \in O^{p^{\prime}}\left(\bar{G}_{\sigma}\right)$ normalizing, but not centralizing $\langle x\rangle$. Therefore $x$ is conjugate to its nontrivial power, a contradiction with Lemma 2.2.

Either $m \geqslant 1$ or $|x|=2$. Assume first that $m \geqslant 1$ and $|x|>2$. Then $Z(C)=S$ and $G_{1} * \ldots * G_{m}$ are normal subgroups of $\langle K, C\rangle$. Hence we may consider $\widetilde{G}=\left\langle K, G_{1} * \ldots * G_{m} * S\right\rangle / S$. Then $\widetilde{G}=\widetilde{K}\left(\mathbf{P} G_{1} \times \ldots \times \mathbf{P} G_{m}\right)$, where $\widetilde{K}=K Z(C) / Z(C)$ is a Carter subgroup of $\widetilde{G}$ (cf. Lemma 2.1) and $Z\left(\mathbf{P} G_{i}\right)$ is trivial. Now $\widetilde{K}$ acts by conjugation on $\left\{\mathbf{P} G_{1}, \ldots, \mathbf{P} G_{m}\right\}$ and without lost we may assume that $\left\{\mathbf{P} G_{1}, \ldots, \mathbf{P} G_{m}\right\}$ is a $\widetilde{K}$-orbit. Thus we are in the condition of Lemma 
2.5 and $\operatorname{Aut}_{\widetilde{K}}\left(\mathbf{P} G_{1}\right)$ is a Carter subgroup of $\operatorname{Aut}_{\widetilde{G}}\left(\mathbf{P} G_{1}\right)$. Moreover $\left|\widetilde{K} \cap \mathbf{P} G_{1} \times \ldots \times \mathbf{P} G_{m}\right|$ is not divisible by the characteristic. By induction we have that $\operatorname{Aut}_{\widetilde{K}}\left(\mathbf{P} G_{1}\right)$ contains a Sylow 2subgroup of $\operatorname{Aut}_{\widetilde{G}}\left(\mathbf{P} G_{1}\right)$, hence the characteristic is odd and $|K \cap G|$ is divisible by 2 . Therefore we may assume $x \in Z(K) \cap K_{G}$ to be an involution. Furthermore, Lemma 3.1 implies that $\left|K_{G}:\left(K_{G} \cap C\right)\right|$ divides $\left|C_{G}(x) / C\right|$ and $C_{G}(x) / C$ is a 2-group.

Thus $x$ is an involution. By Lemma 3.5 we have that every involution of $G$ is contained in a maximal torus $T$ such that $N(G, T) / T \simeq W$, where $W$ is the Weyl group of $\bar{G}$. In particular, we may assume that $\bar{C}$ is generated by $\bar{T}$-invariant root subgroups and that $\zeta_{2^{\prime}}$ centralizes $C_{G}(x) / C$ (by [23, Proposition 2] $N(G, C) / C \simeq N_{W}\left(W_{1}\right) / W_{1}$, where $W=N(G, T) / T$ and $\left.W_{1}=N(C, T) / T\right)$. In view of Lemma 4.3, $\zeta_{2^{\prime}}$ normalizes $T$, each of $G_{i}$ and centralizes some Sylow 2-subgroups of $T$ and of each of $G_{i}$. We can write $\zeta g=\zeta_{2} g_{1} \cdot \zeta_{2^{\prime}} g_{2}$, where $\zeta_{2} g_{1}$ is a 2-part and $\zeta_{2^{\prime}} g_{2}$ is a $2^{\prime}$-part of $\zeta g$. Since $\zeta_{2^{\prime}}$ centralizes $C_{G}(x) / C$ and since $C_{G}(x) / C$ is a 2-group, we obtain that $g_{2} \in C$. Hence $\zeta_{2^{\prime}} g_{2}$ normalizes $T$ and each of $G_{i}$. As we noted above, $\zeta_{2^{\prime}}$ centralizes a Sylow 2-subgroup of $T$, hence, it centralizes a Sylow 2-subgroup of $Z(C) \leq T$. Thus we have that every element of odd order of $\left\langle K, C_{G}(x)\right\rangle$ centralizes the Sylow 2-subgroup of $Z(C)$.

Now consider $\widetilde{G}=\langle K, C\rangle / Z(C)$. Then $\widetilde{G}=\widetilde{K}\left(\mathbf{P} G_{1} \times \ldots \times \mathbf{P} G_{m}\right)$ (possibly $m=0$ ), where $\widetilde{K}=K Z(C) / Z(C)$ is a Carter subgroup of $\widetilde{G}$ (cf. Lemma 2.1) and for all $i, Z\left(\mathbf{P} G_{i}\right)=1$. By Lemma 2.5 we have that $\operatorname{Aut}_{\widetilde{K}}\left(\mathbf{P} G_{1}\right)$ is a Carter subgroup of $\operatorname{Aut}_{\widetilde{G}}\left(\mathbf{P} G_{1}\right)$. Since $\mathbf{P} G_{1}$ is a finite group of Lie type satisfying Theorem 7.1 , by induction we obtain that $\operatorname{Aut}_{\widetilde{K}}\left(\mathbf{P} G_{1}\right)$ contains a Sylow 2-subgroup of $\operatorname{Aut}_{\widetilde{G}}\left(\mathbf{P} G_{1}\right)$. Similarly we have that $\operatorname{Aut}_{\widetilde{K}}\left(\mathbf{P} G_{i}\right)$ contains a Sylow 2-subgroup of $\operatorname{Aut}_{\widetilde{G}}\left(\mathbf{P} G_{i}\right)$ for all $i$. Let $S$ be a Sylow 2-subgroup of $N_{\widetilde{G}}\left(\mathbf{P} G_{1}\right)$. Since $C_{\widetilde{G}}\left(\mathbf{P} G_{1} \times \ldots \times \mathbf{P} G_{m}\right)=\{1\}$, Lemma 2.4 implies that $N_{N_{\widetilde{G}}\left(\mathbf{P} G_{1}\right)}(S)=S C_{N_{\widetilde{G}}\left(\mathbf{P} G_{1}\right)}(S)$. Now $\left|\widetilde{G}: N_{\widetilde{G}}\left(\mathbf{P} G_{1}\right)\right|=2^{t}$, thus, by Lemma 2.6, we obtain that $N_{\widetilde{G}}(\widetilde{S})=\widetilde{S} C_{N_{\widetilde{G}}\left(\mathbf{P} G_{1}\right)}(\widetilde{S})$ for a Sylow 2-subgroup $\widetilde{S}$ of $\widetilde{G}$. Since $\left|\mathbf{P} G_{i}\right|<C$ min, then $\widetilde{G}$ and $\langle K, C\rangle$ satisfy $(*)$. By Lemma 2.3 we obtain that there exists a Carter subgroup $M$ of $\langle K, C\rangle$ that contains a Sylow 2-subgroup of $\langle K, C\rangle$. By Theorem 1.1, subgroups $M$ and $K$ are conjugate in $\langle K, C\rangle$, thus $K$ contains a Sylow 2-subgroup $Q$ of $\left\langle K, C_{G}(x)\right\rangle$.

Let $S_{1}$ be a Sylow 2-subgroup of $\Gamma G$ containing $Q$ and let $t \in Z\left(S_{1}\right) \cap G$. Then $t \in C_{G}(x)$, hence, $t \in Z(Q)$ and $t \in Z(K)$. Thus we may substitute $x$ by $t$ in arguments above and obtain that $Q=S_{1}$, i. e., $K$ contains a Sylow 2-subgroup of $\Gamma G$.

\section{Carter subgroups of finite groups are conjugate}

In order to state the following theorem without using the classification of finite simple groups, we give the following definition. A finite group is said to be a $K$-group if all its non-Abelian composition factors are known simple groups.

Theorem 10.1. Let $G$ be a finite $K$-group. Then Carter subgroups of $G$ are conjugate.

Proof. By [10, Theorems 3.3-3.5], [6, Theorem 1.1], [9, Table]; and Theorems 5.1, 6.1, and 7.1 from the present paper we obtain that $G$ satisfies (*). Hence, by Theorem 1.1, Carter subgroups of $G$ are conjugate.

\section{References}

[1] R. W. Carter, Nilpotent self-normalizing subgroups of soluble groups, Math. Z., 75 (1961), $136-139$. 
[2] L. Di Martino and M.C. Tamburini, I sottogruppi nilpotenti autonormalizzanti di $S_{n}$ e di $A_{n}$, Istit. Lombardo Accad. Sci. Lett. Rend. A, 110 (1976), 235-241.

[3] L. Di Martino and M. C. Tamburini, Carter subgroups of projective linear groups, Boll. Un. Mat. Ital. B, 7 (1987), 905-915.

[4] N. A. Vavilov, Nilpotent self-normalizing subgroups of the general linear group over a finite field, Zap. nauchn. Sem. Leningrad Otdel Mat. Inst. Steklov LOMI, 86 (1979), 34-39.

[5] L. Di Martino, M. C. Tamburini and A. E. Zalesskii, Carter subgroups in classical groups, J. London Math. Soc. (2), 55 (1997), 264-276.

[6] A. Previtali, M. C. Tamburini, E. P. Vdovin, The Carter subgroups of some classical groups, Bull. London Math. Soc., 36, N 1 (2004), 145-155.

[7] A. D'aniello, Sull' esistenza di sottogruppi nilpotenti autonormalizzanti in alcuni gruppi semplici, II, Atti Accad. Naz. Lincei Rend. Cl. Sci. Fis. Mat. Natur., 74 (1983), 1-6.

[8] F. Dalla Volta, A. Lucchini, and M. C. Tamburini, On the Conjugacy Problem for Carter Subgroups, Comm. Algebra, 26, N 2 (1998), 395-401.

[9] E. P. Vdovin, On the conjugacy problem for Carter subgroups, SMJ, to appear. (see also http://arxiv.org/abs/math/0602125)

[10] M. C. Tamburini, E. P. Vdovin, Carter subgroups of finite groups, J.Algebra, 255, N 1 (2002), 148-163.

[11] W. Feit, J. Thompson, Solvability of groups of odd order, Pacif. J. Math., 13, N 3 (1963), 775-1029.

[12] R. W. Carter, Simple groups of Lie type, John Wiley and Sons, 1972.

[13] J. E. Humphreys, Linear algebraic groups, Springer-Verlag, New York, 1972.

[14] R. Steinberg, Automorphisms of finite linear groups, Canad.J.Math., 12, N 4 (1960), 606615.

[15] R. W. Carter, Finite groups of Lie type, conjugacy classes and complex characters, John Wiley and Sons, 1985.

[16] $R$. W. Carter, Centralizers of semisimple elements in the finite classical groups, Proc. London Math. Soc. (3), 42, N 1 (1981), 1-41.

[17] D. Deriziotis, Conjugacy classes and centralizers of semisimple elements in finite groups of Lie type, Vorlesungen aus dem Fachbereich Mathematic der Universität Essen, 111984.

[18] R. Steinberg, Endomorphisms of algebraic groups, Mem.AMS, 80, 1968.

[19] R. Steinberg, Lectures on Chevalley groups, Yale University, 1967.

[20] A. Borel and J. de Siebental, Les-sous-groupes fermés de rang maximum des groupes de Lie clos, Comment.Math.Helv., 23 (1949), 200-221. 
[21] E. B. Dynkin, Semisimple subalgebras of semisimple Lie algebras, Mat.sb., 30, N 2 (1952), 349-462. (In russian)

[22] J. E. Humphreys, Conjugacy classes in semisimple algebraic groups, American Mathematical Society, Providence, Rhode Island, Mathematical Survey and Monographs, 43, 1995.

[23] $R$. W. Carter, Centralizers of semisimple elements in finite groups of Lie type, Proc. London Math. Soc. (3), 37, N 3 (1978), 491-507.

[24] A. S. Kondratiev, Normalizers of Sylow 2-subgroups in finite simple groups, Math.Notes, 78, N 3-4 (2005), 338-346.

[25] A. Borel, R. Carter, C. W. Curtis, N. Iwahori, T. A. Springer, R. Steinberg, Seminar on algebraic groups and related finite groups, Springer-Verlag, 1970.

[26] R. W. Carter, Conjugacy classes in the Weyl group, Compositio Mathematica, 25, N 1 (1972), 1-59.

[27] B. Huppert, Endliche Gruppen. I, Springer, Berlin, 1967.

[28] W.Feit and G.Zukerman, Reality properties of conjugacy classes in spin groups and symplectic groups, Algebraists' homage:papers in ring theory and related topics, New Haven, 1981, 239-253.

[29] D. Gorenstein, R. Lyons, The local structure of finite groups of characteristic 2 type, Mem. AMS, 42 (1983), ISSN 0065-9266.

[30] H. N. Ward, On Ree's series of simple groups, Trans. Amer. Math. Soc., 121, N 1 (1966), $62-80$.

[31] V. M. Levchuk, Ja. N. Nuzhin, Structure of Ree groups, Algebra and Logic, 24, N 1 (1985), 16-26.

[32] J. H. Conway, R. T. Curtis, S. P. Norton, R. A. Parker, and R. A. Wilson, Atlas of finite groups, Clarendon Press, Oxford (1985).

[33] The GAP Group, GAP — Groups, Algorithms, and Programming, Version 4.4 (2004); (http://www.gap-system.org).

[34] M. J. Wonenburger, Transformations which are products of involutions, J.Math.Mech., 16, N 1 (1966), 32-38???

[35] G. M. Seitz, Unipotent elements, tilting modules, and saturation, Inv. Math, 141, N 3 (2000), 467-503.

[36] P. H. Tiep, A. E. Zalesski, Real conjugacy classes in algebraic groups and finite groups of Lie type, J.Group Theory, 8, N 3 (2005), 291-315. 